\begin{tabular}{|c|l|}
\hline Title & $\begin{array}{l}\text { A METHOD OF STATISTICAL MECHANICAL TREATMENT OF EQUILIBRIUM AND CHEMICAL } \\
\text { REACTIONS. }\end{array}$ \\
\hline Author(s) & HORIUTI, Juro \\
\hline Citation & JOURNAL OF THE RESEARCH INSTITUTE FOR CA TALY SIS HOKKA IDO UNIVERSITY, 1(1), 8.79 \\
\hline Issue Date & 194811 \\
\hline Doc URL & http://hdl.handle.net/2115/24601 \\
\hline Type & bulletin (article) \\
\hline File Information & 1(1)_P8 79.pdf \\
\hline
\end{tabular}

Instructions for use 


\title{
A METHOD OF STATISTICAL MECHANICAL TREATMENT OF EQUILIBRIUM AND CHEMICAL REACTIONS.
}

\author{
By Juro Honiuti. \\ The Research Institute for Catalysis, Hokkaido Imperial University Sapporo, Hokkaido. \\ Reeeived 15 March, 1945.
}

\section{INTRODUCTION}

The present paper is concerned with a method of treating equilibrium and chemical reaction by introducing a set of conjugated statistical mechanical functions defined respectively as the factor of multiplication of the Zustandsumme caused by increasing a certain constituent of the assembly of interest or by imposing a microscopic constraint upon the elementary state of the constituent.

- Okamoto, Horiuti and Hirota ${ }^{(1)}$ have previously introduced the functions $p^{\tilde{\sigma}}$ and $q_{\sigma}^{\delta}$ of this sort in their statistical mechanical treatment of the hydrogen electrode process. Among these $p^{\hat{\sigma}}$ is relaten, as shown later, to the chemical potential $\mu^{\hbar}$, as that,

$$
\mu^{\delta}=-R T \log p^{\delta}
$$

which was originally introduced by Gibbs $^{(2)}$ in terms of classical grand canonical ensemble, whereas $p^{\delta}$ is defined here, according to Gibbs' terminology, in terms of petit canonical ensemble. The function is introduced in the latter way independently by Kirkwood ${ }^{(3)}$ and applied to the statistical mechanics of the condensed fluid in equilibrium. The function $q_{\sigma}^{\delta}$ has, on the other hand, no corresponding thermodynamical quantity. In the present paper we are going to introduce, in extention of the

(1) Okamoto, Horiuti and Hirota; Sc. Pap. I.P.C.R. Tokio, 29 (1936), 228.

(2) Gibbs; Collected Works, 2, Part 1, "Elementary Principles in Statistical Mechanics." Longmans, (1928).

(3) Kirkwood ; J. Chem. Phys. 3 (1935), 300. "Statistical Mechanies of Fluid Mixture." Chem. Rev. 19 (1936), 275. "Statistical Mechanies of Liquid Solutiona." 
A Method of Statistical Mechanical Treatment of Equilibrium and Chemical Reactions.

theory of $p^{6}$ and $q_{\sigma}^{\delta}$, a complete set of four conjugated functions and give several examples of application of the equilibrium and cherrical reaction:

\section{CHAPTER I \\ DEFINITIONS AND APPROXIMATIONS}

\section{§. The Assembly and Parameters.}

Our object of treatment will be a macroscopic assembly composed of molecules of several kinds, whose set of quantum mechanical Eigenwerts being fixed by several independent parameters. The parameters may be the volume confining the total extension of the assembly, the electrostatical or magnetic field within which the whole assembly is situated. These parameters which are quantities accesible to the direct macroscopic measurements will specially be termed external ones in distinction from internal parameters later referred to, which, being inaccesible to direct macroscopic measurements, restrict microscopic states of individual molecules. The macroscopic assembly will be denoted by $C$ in general.

The assembly will be termed in statistical equilibrium when its properties are derivable from the appropriate petit quantum canonical ensemble. The Zustandsumme (abbreviated $\mathrm{Zs}$ hereafter) appropriate to any equilibrium state of $C$ is expressed by

$$
\mathfrak{O C}=\sum_{n} e^{-\frac{E_{n}}{k T}}
$$

where $k$ is Boltzmann's constant and $E_{n}$ the $n$-th Eigenwert.

The molecule in the above sense may consist in any elementary particle or any group of elementary particles bound closely together, however, not being necessarily dynamically isolated from the environment. By molecule will thus be meant hereafter not only gaseous molecule in its original sense, but also such an elementary particle or a group of elementary particles in a liquid phase under the strong influence of surrounding ones or even such one bound chemically on the surface of a solid.

\$2. Four Conjügated Functions, $p^{\delta}, q_{\sigma}^{\delta}, \boldsymbol{\theta}_{\sigma(\delta)}$ and $\boldsymbol{\theta}_{o(0)}$.

We define $\dot{p}^{\delta}$ by, 


$$
p^{\delta}=\frac{\mathfrak{Q} C_{0}^{8}}{\mathfrak{Q} C_{0}^{\prime}}
$$

where $\mathfrak{Q} C_{0}$ is $\mathrm{Zs}$ of our assembly $C$ free, in particular, from any microscopic constraints, and $\mathfrak{Q C}_{0}^{\delta}$ that of $C_{0}^{\delta}$, which is $C$ augmented by a molecule or a set of molecules $\delta$ with fixed external parameters.

The function $q_{\mathrm{o}}^{\mathrm{d}}$ is defined by,

$$
q_{\sigma}^{\delta}=\frac{\mathfrak{Q} C_{\sigma(\delta)}^{\delta}}{\mathfrak{Q} C_{o(0)}^{\delta}}
$$

where $S C_{\sigma(\delta)}^{\delta}$ or $S C_{\sigma(0)}$ is $\mathrm{Zs}$ of the assembly $C_{\sigma(\hat{\sigma})}^{\delta}$ or $C_{o(0)}$ respectively subject to the microscopic constraint, i.e. a particular elementary state $\sigma$ for a single piece of molecule $\delta$ or a set $\sigma$ of states each for individual piece of a set $\delta$ of molecules is occupied by $\delta$ or vacant. The elementary state $\sigma$ or the individual state of the set $\sigma$ may be a small cavity of molecular dimension confining the centre of gravity of a molecule or a certain quanturn state for the energy or the momentum of the latter.

We define further two quantities in terms of the Zs's appeared in Eqs. (2.1) and (2.2) as that,

$$
\boldsymbol{\theta}_{o(\delta)}=\frac{\mathfrak{Q} C_{\sigma(\delta)}^{\delta}}{\mathfrak{Q} C_{o}^{\delta}}
$$

and that,

$$
\boldsymbol{\theta}_{\sigma(0)}=\frac{\mathfrak{D} C_{\boldsymbol{\sigma}(0)}}{\mathfrak{D} C_{0}}
$$

$\boldsymbol{\theta}_{\sigma(\delta)}$ or $\boldsymbol{\theta}_{\sigma(0)}$ has thus the physical meaning of the respective probability that $\sigma$ is occupied or evacuated by $\delta$.

\section{§. Extension of the Definitions.}

The above defined functions may also be termed the respective factors of multiplication of $\mathrm{Zs}$ caused by the appropriate operations; $p^{\delta}$ is thus the factor due to the operation of adding $\delta$ to the assembly $C_{0}$ with unconstrained internal parameters bringing about thereby an assembly $C_{0}^{\delta}$ of the same description but with one more constituent molecule $\delta$, $q_{\sigma}^{*}$ that of bringing one additional $\delta$ up into vacant $\sigma$ of $C_{o(0)}$ and $\boldsymbol{\theta}_{\sigma(\hat{\sigma})}$ or $\theta_{o(0)}$ that of imposing the constraint upon the unconstrained assembly 
$C_{6}^{6}$ or $C_{0}$ that a specified state $\sigma$ is respectively occupied by $\delta$ or evacuated with certainty.

With this interpretation above definitions may be expressed in the forms,

$$
\begin{aligned}
& \log p^{\delta}=\mathcal{A}_{p_{,} \delta} \log \mathfrak{\Omega C} C_{0} \\
& \log q_{\sigma}^{\delta}=\mathcal{u}_{q, \sigma(0)} \log \mathfrak{D} C_{o(0)} \\
& \log \boldsymbol{\theta}_{\sigma(\delta)}=\Delta_{\theta_{1} \sigma(\delta)} \log \mathfrak{Q} C_{\theta}^{\delta} \\
& \log \boldsymbol{\theta}_{\sigma(0)}=A_{\theta, \sigma(0)} \log \Omega C_{0}
\end{aligned}
$$

or summerized as that,

$$
\log \lambda=\Delta_{\lambda} \log \Omega C^{\prime}
$$

where $\lambda$ stands for $p^{\delta}, q_{\sigma}^{\delta}, \boldsymbol{\theta}_{\sigma(\delta)}$ or $\boldsymbol{\theta}_{\sigma(0)}$ and $\boldsymbol{\Delta}_{\lambda}$, when prefixed for instance to $\log \mathfrak{\Omega} C$, gives the increment caused by the operation appropriate to $\lambda$. For the sake of having any meaning for the quantity with the prefix, the assembiy of interest must however be accessible to the operation associated with $\lambda$. It is meant by "accessible," for instance, to the operation associated with $q_{\sigma}^{\delta}$ that the assembly $C_{\sigma(0)}, C_{\sigma(0)}^{\delta}$ or such other has $\sigma$ free to accept $\delta$ or to the operation associated with $\boldsymbol{\theta}_{o(\delta)}$ that $C_{0}$, $C^{s}$ are, not being constrained by any prescribed condition, ready to access the operation of filling up $\sigma$ with $\delta$ with certainty.

We define $d_{\lambda} P$ as the increment of any property $P$ accompanied by the operation $J_{\lambda}$ upon the assembly, and the quantity prefixed with $s_{p, \lambda}$ for instance $\Delta_{p, \lambda} \log \mathfrak{Q C}$ as that of $\log \mathfrak{Q C}$ caused by increasing $P$ alone by the amount $\Delta_{\lambda} P$ without virtual operation.

These increment defined above will be called first order increments. Denoting any two of these $A_{\text {'s }}$ by $\Delta_{1}$ and $\Delta_{2}$ we define second order increment $\Delta_{1} \Delta_{2} \log \mathfrak{Q C} \equiv \Delta_{1} \log \mathfrak{Q C}\left(\Delta_{2}\right)-J_{1} \log \Omega C$ and $\Delta_{1} \Delta_{2} P \equiv \Delta_{1} P\left(\Delta_{2}\right)-\Delta_{1} P$, where $C\left(d_{2}\right)$ is the assembly brought about from $C$ by the operation associated with $A_{2}$ and $P\left(A_{2}\right)$ the appropriate value of $P$, it being presupposed that both $C$ and $C\left(A_{2}\right)$ are accessible to the operation $A_{1}$. Similarly we define higher order increments.

\section{\$4. Approximations.}

Throughout the present paper we will be contented with the appro- 
ximation of negiecting increments of higher than second order or power compared with the increment of first order or power except when the direct interaction between two molecules each at an elementary state $\sigma_{1}$ or $\sigma_{2}$ respectively specified, if at all, by the two operations involved, such as in the case when $A_{1} \equiv d_{\theta, \sigma_{1}(\delta)}$ and $d_{2} \equiv d_{\theta, \sigma_{2}(\delta)}$, is significant. If either or both of the operation are of the sort $J_{p_{0},}$, which specifies no elementary state, this exception falls of course off.

It follows that,

$$
J_{p, \delta_{2}}\left(\log \subseteq C_{0}+J_{p, \Sigma_{1}} \log \subseteq C_{0}\right)=d_{p, \delta_{2}} \log \cong C_{0}
$$

But since according to Eqs. (3.1) and (2.1), it is that,

$$
\log \subseteq C_{0}+\Delta_{p, \delta_{1}} \log \mathfrak{Q} C_{0}=\log \mathfrak{Q} C_{0}^{d_{1}}
$$

we have,

$$
\frac{\mathfrak{Q} C_{0^{1}}^{\delta_{1}+\delta_{2}}}{\mathfrak{Q} C_{0_{1}^{1}}^{\delta}}=\frac{\mathfrak{Q} C_{0^{\prime}}^{\delta}}{\mathfrak{Q} C_{0}}
$$

Writing $p^{\delta_{1}+\varepsilon_{2}}$ for the set of molecules $\delta_{1}+\delta_{2}$ according to Eq. (2.1) in the form,

$$
p^{\delta_{1}+\delta_{2}}=\frac{\mathfrak{Q} C_{0}^{\delta_{1}+i_{2}}}{\mathfrak{Q} C_{0}}=\frac{\mathfrak{D} C_{0}^{\delta_{1}+\delta_{2}}}{\mathfrak{Q} C_{0}^{\delta_{1}}} \cdot \frac{\mathfrak{Q} C_{0}^{\delta_{1}}}{\mathfrak{D C} C_{0}}
$$

We have,

$$
p^{\hat{\alpha}_{1}+\hat{\ell}_{2}}=p^{\delta_{1}} \cdot p^{\delta_{2}}
$$

It may similarly be shown for a set $\delta$ of several molecules,

$$
\delta=\sum_{0} \psi_{D} \delta_{g}
$$

consisting each $\nu_{g}$ pieces of $\delta_{g}$, that

$$
p^{\delta}=\stackrel{o}{I}\left(p^{\delta} \sigma\right)^{v_{o}}
$$

(ii) We have similarly as in the case of (i), that,

$$
\Delta_{\theta, 0(8)}\left(\log \Omega C_{0}+J_{p} \log \Omega C_{0}\right)=\Delta_{\theta, 0(8)} \log \Omega C_{e}
$$

or according to Eqs. (3.3) and (2.3), that, 


$$
\boldsymbol{\theta}_{\sigma(\delta)}=\frac{\mathfrak{S} C_{o(\delta)}^{\delta}}{\mathfrak{S} C_{0}^{\delta}}=\frac{\mathfrak{S} C_{o(\delta)}}{\Omega C_{0}}
$$

(iii) Any increment of $\log \mathfrak{\Omega} C$ may be written in the form,

$$
\Delta_{\lambda} \log \mathfrak{Q} C=\left(\Delta_{\lambda} \log \mathfrak{Q} C\right)_{P}+\sum_{j} \Delta_{P_{j^{\prime}}} \log \mathfrak{Q} C
$$

where the $\left(\Delta_{\lambda} \log \Omega C\right)_{p}$ denotes the increment in the case when all properties $\boldsymbol{P}_{\boldsymbol{j}}$ 's of interest are arrested. Expanding $\Delta_{p_{j \cdot \lambda}} \log \mathfrak{Q C}$ in power series of $\boldsymbol{S}_{\lambda} \boldsymbol{P}_{\text {; }}$ and rejecting terms higher than the first power, we have,

and hence

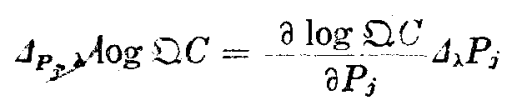

$$
1 \quad \Delta_{\lambda} \log \mathfrak{Q C}=\left(\Delta_{\lambda} \log \mathfrak{Q} C\right)_{P}+\sum_{j} \frac{\partial \log \mathfrak{Q} C}{\partial P_{j}} d_{\lambda} P_{j}
$$

\section{CHAPTER II.}

\section{THEORY.}

\$5. Interrelation among $p^{\delta}, q_{\sigma}^{\delta}, \Theta_{\sigma(\delta)}$ and $\Theta_{\sigma(0)}$.

It follows immediately from Eqs. (2.1), (2.2), (2.3) and (2.4) that,

$$
q_{\mathrm{c}}^{\delta}=\frac{\boldsymbol{\theta}_{\tau(\vartheta)}}{\boldsymbol{\theta}_{\sigma(0)}} p^{\delta}
$$

If $\delta$ consists in a single molecule and $\sigma$ a small cavity of molecular dimension which restricts the centre of gravity of $\delta$ within, the above equation rnay be written in the form,

$$
p^{\delta}=\boldsymbol{\theta}_{o(0)} \frac{q_{\sigma}^{\delta} / ! \sigma !}{\boldsymbol{\theta}_{\sigma(\delta)} / \mid \sigma !}
$$

where $|\sigma|$ is the size of the cavity $\sigma$, As $|\sigma|$ approaches zero, so does $\boldsymbol{\theta}_{\sigma(0)}$ unity and $\frac{\theta_{\sigma(\delta)}}{|\sigma|}$ the limiting ratio of the probability of finding the centre of gravity of the molecule of the kind $\delta$ in the cavity to its volume or the concentration $N^{\delta}$ at the point of convergence, i.e. 


$$
N^{\bar{\delta}}=\operatorname{Lim}_{|\bar{\sigma}| \rightarrow 0} \frac{\theta_{\sigma(\delta)}}{|\sigma|}
$$

Since $p^{\delta}$ should remain the same by definition irrespective of the arbitrarily assigned magnitude $|\sigma|, q_{\sigma}^{\delta} /|\sigma|$ tends to a definite value along with $\theta_{\sigma(i, j}|| \sigma \mid$. Denoting the limiting value by $Q^{\delta}$, i.e. putting

$$
\lim _{\mid a \rightarrow 0} \frac{q_{o}^{\delta}}{|\sigma|}=Q^{\delta}
$$

We have Eq. (2.1) in the form,

$$
p^{\delta}=\frac{Q^{\delta}}{N^{\delta}}
$$

\$6. Equilibrium Relation for $p^{\delta}$.

Let the condition prescribed to the assembly be such that $\delta$ may exist in different states, $\delta^{A}, \delta^{B}, \ldots$ etc., $\Omega C^{\delta^{A}}, \mathfrak{\Omega C} \boldsymbol{\delta}^{B}$ etc. are now of the same value, since it is immaterial for the $Z$ s over all possible states of the assembly, whatever state $\delta$ may initially assume when admitted. It follows by $\mathrm{Eq}$. (2.1) that,

$$
p^{\hat{\delta}^{A}}=p^{\delta^{B}}=\text { etc. }
$$

The states $\delta^{A}, \delta^{B}$ etc. will be called in equilibrium with each other.

If $\delta^{A}, \delta^{B}$ etc. are respectively such sets of molecules as that,

$$
\delta^{A}=\sum_{a} \nu_{a}^{A} \delta_{a}^{A} \quad \delta^{B}=\sum_{b} \nu_{b}^{B} \delta_{b}^{B}
$$

i.e. consist respectively of $\nu_{a}^{A}, \nu_{b}^{B}$ ete. pieces of individual molecules $\delta_{a}^{A}, \delta_{b}^{B}$, ete., we have by Eqs. (4.1) and (6.1) that,

$$
\stackrel{a}{I}\left(p^{\delta_{a}^{A}}\right)^{A} A \stackrel{b}{I}\left(\chi^{\delta} \delta_{b}^{B}\right) \nu_{b}^{B}
$$

\$7. Force, Work and Energy.

We postulate that there exists a set of internal parameters such that by varying the latters continuously independent of each other and of the external ones, the microscopic state of constituent molecules is transferred from one to the other keeping the whole assembly at every 
instant at the statistical equilibrium. The assembly $C_{0}$ may thus be converted into $C_{\sigma(\delta)}$ or $C_{\sigma(0)}$ and the assembly consisting of macroscopic assembly $C_{0}$ or $C_{\sigma(0)}$ and a molecule $\delta$ each situated outside the influence of the other, i.e. $C_{0}+\delta$ or $C_{\mathrm{o}(0)}+\delta$ may thus be unified into $C_{\mathrm{o}}^{\delta}$ or $C_{\mathrm{o}(0)}^{\delta}$. All parameters including internal and external ones will be denoted by $\boldsymbol{a}_{a}$ 's. We have then according to Eq. (1.1),

where

$$
-k T \log \frac{\mathfrak{Q} C_{I I}}{\mathfrak{Q} C_{I}}=\sum_{\boldsymbol{a}} \int_{\alpha_{a}(I)}^{\boldsymbol{x}_{a}(I I)}\left(-I I_{\alpha_{a}}\right) \cdot d \alpha_{\boldsymbol{a}}
$$

$$
I I_{\alpha_{a}}=\frac{\partial(k T \log \Omega C)}{\partial \alpha_{a}}=\frac{\sum_{n}-\frac{\partial E_{n}}{\partial \alpha_{a}} e^{\cdot \frac{F_{n}}{k T}}}{\sum_{n} e^{-\frac{E_{23}}{k T}}}
$$

Suffix $I$ and $I I$ signify two different particular state of our assembly, and $\alpha_{a}(I)$ and $\alpha_{a}(I I)$ appropriate values of the parameters $\alpha_{a}$. The partial differential coeficient $-\frac{\partial E_{n}}{\partial \sigma_{a}}$ is the force with which the assembly at $n$ th quantum state tends to increase with the paramets $\alpha_{a}, I_{\alpha_{n}}$ the statistical average of the foree, $-I I_{\alpha_{a}}$ the force to be applied from the external world to the assembly to keep $\alpha_{a}$ constant, and, in consequence the left hand side of Eq. (7.1) the work required to bring up the assembly from the state $I$ to $I I$ keeping the latter throughout at st tistical equilibrium. The $I_{a_{a}}$ will be called the average force coajugated to the parameter $\alpha_{a}$ and the work the reversible work.

In the particular case when

$$
C_{I}=C_{0}+\delta \text { and } C_{I I}=C_{0}^{\delta}
$$

the left hand side of Eq. (7.1) is $-k T \log p^{\delta},{ }^{(*)}$ which equals the relevant reversible work on the right. The function $-k T \log q_{a}^{s}$ is similarly the reversible work required with fixed external parameters of $\left({ }_{o}()\right.$ to bring up $\delta$ from the standard state into the preliminarily evacuat a state $\sigma$, and $-k T \log \theta_{\sigma(0)}$ or $-k T \log \theta_{\sigma(\delta)}$ that required to force upon the assembly $C_{0}$ the constraint that $\sigma$ is respectively vacunt or occupied by $\delta$ with

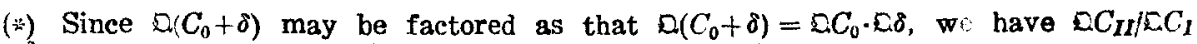
$=\Omega C_{0}^{\delta} / \Sigma C_{0}$ by choosing that $\Omega \delta=1$. 
certainty. The latter three reversible works naturally correspond to no ready-made thermodynamical quantity because of its microscopically detailed nature.

These reversible works $-k T \log p^{\delta},-k T \log q_{\sigma}^{\delta},-k T \log \theta_{\sigma(\delta)}$ and $-k T \log \theta_{a(0)}$ are summarized, according to $\mathrm{Eq}$. (3.5), by,

or by

$$
-k T \log \lambda=\Delta_{\lambda}(-k T \log \mathfrak{D C})
$$

$$
-R T \log \lambda=\left.\Delta\right|_{\lambda}(-k T \log \mathfrak{D C})
$$

where $\|_{\lambda}$ signifies Avogadro's number $N_{A}$ times the increment $\boldsymbol{d}_{\lambda}$.

The temperature variation of $\mathfrak{Q} C$ at fixed external parameter $a_{a}^{\prime}$ 's is as well known expressed according to Eq. (1.1) in terms of statistical average $\bar{E}$ of Eigenwerts, i.e.,

$$
k T^{2} \frac{\partial \log \mathfrak{Q} C}{\partial T}=\frac{\sum_{n} E_{n} e^{-\frac{E_{n}}{k T}}}{\sum_{n} e^{-\frac{E_{n}}{k} T}}=\bar{E}
$$

Partial differential coefficients with respect to $T$ without suffix will hereafter be referred to fixed external parameters. The temperature variation of $\lambda$ is henceforth expressed according to Eqs. (7.4) and (7.5), as that,

$$
R T^{2} \frac{\partial \log \lambda}{\partial T}=\Delta \mathbb{L}_{\lambda} \bar{E}
$$

\$. The Functions and Associated Relations in Thermodymamical Terminology.

Provided that the statistical equilikrium fully describes the thermodynamical equilibrium, $-k T \log \mathfrak{Q} C$ may be identified with Helmholtz free energy $F$ according to $\mathrm{Eq} .(7.1)$ and $\bar{E}$ with the internal energy $U$ in thermodynamics,

so that

and

$$
\begin{aligned}
& F=-k T \log \mathfrak{2} C \\
& U=k T^{2} \frac{\partial \log \mathfrak{D} C}{\partial T}
\end{aligned}
$$

We have immediately according to Eqs. (8.1) and (8.2) that, 
A Method of Statistical Mechanical Treatment of Equilibrium and Chemical Reacions.

$$
U=F-T \frac{\partial F}{\partial T}
$$

from Eu. $(7,2) \quad-I I_{\alpha_{a}}=\frac{\partial F}{\partial r_{a}}$

from $\mathrm{Eq} .(7.4)$

$$
\Delta l_{\lambda} F=-R T \log \lambda
$$

and from Eq. (7.6)

$$
\left.\Delta\right|_{\lambda} U=R T^{2} \frac{\partial \log \lambda}{\partial T}
$$

or from Eq. (8.3), $\left.\quad \Delta\right|_{\lambda} U=\left.\Delta\right|_{\lambda} F-T \frac{\partial \Delta l_{\lambda} F}{\partial T}$

Defining the entropy to complete the thermodynamical terminology as that,

$$
S=-\frac{\partial F^{(*)}}{\partial T}
$$

we have readily,

$$
\Delta \|_{\lambda} S=-\frac{\left.\partial \Delta\right|_{\lambda} F}{\partial T}
$$

and from Eq. (8.3),

or

$$
\begin{aligned}
& F=U-T S \\
& \Delta\left\|_{\lambda} F=\Delta\right\|_{\lambda} U-T \Delta_{\lambda} S
\end{aligned}
$$

In the special case when $\lambda=p^{\delta}$, the reversible work $-R T \log p^{\delta}$ may be identified with the chemical potential $\mu^{\delta}$ in thermodynamics so that,

$$
\mu^{\delta}=-R T \log p^{\delta}
$$

Fiqs. (8.5), (8.6), (8.9) and (8.11) then assume respectively the forms, i.e.,

(*) The presont argument is evidently valid in particular in the case of microcanonical ensemble, when, all quantum state have the same Eigenwert $E_{n}$. In that case, Eq. (8.1) reduces, according to Eq. (1.1) to the form,

$$
F=E_{n}-k T \log \sum_{n} 1
$$

whenee we have by Eq. (8.8), $S=k \log \sum_{x} 1$. This is the quantum mechanical transcription of the well-known Boltzmann's relation. 


$$
\mu^{\delta}=\bar{F}^{\delta}
$$

$$
\begin{aligned}
& R T^{y} \frac{\partial \log p^{\delta}}{\partial T}=\bar{U}^{\delta} \\
& \bar{U}^{\delta}=\mu^{\delta}-T \frac{\partial \mu^{\delta}}{\partial T}
\end{aligned}
$$

$$
\bar{S}^{\delta}=-\frac{\partial \mu^{\delta}}{\partial T}
$$

$$
\mu^{\delta}=\bar{U}^{\delta}-T \bar{S}^{\delta}
$$

where $\bar{F}^{\delta}, \bar{U}^{\delta}$ and $\bar{S}^{\delta}$ denotes respectively $\Delta_{p, \delta} F, \Delta_{p . \delta} U$ and $\Delta_{p, \delta} S^{(*)}$ and called in accordance with G.N. Lewis partial molal free energy, partial molal internal energy and partial molal entropy resp ctively.

Our approximation (i), $\$ 4$, assumes now tha focm,

$$
\mu^{\delta}=\sum_{g} \nu_{g} \mu^{\delta_{\theta}}
$$

which is taken as a matter of course in thermodynomics.

\section{\$9. The ג for the Assembly with Variable External Parameters.}

In the foregoing section $\lambda$ 's have been $d$ fir $d$ with assembly whose external parameters are kept constnt throughont the operation associated with respective $\lambda$ 's. We extand h re thir deficition and theory to the case when the external parametrs of the assembly of interest are not necessarily kept constant.

Let an assembly of interest $A$, which exclusiv-ly subject to the operation associated with $\lambda$, is coupled with anoth $>r$ according to the condition that (i) each external parameter which is xtansive property such as volume, surface area etc. is not fix in di idually with $A$ and $K$ but by the sum of that, $\beta_{b}$, of $A$ and thet of $K$. whress external parameters of other kind is fixed individually (ii) no for coujugat $\mathrm{c}$ with $\beta_{b}$ 's acts upon $A+K$ from outside and that (iii) enurg states of either assembly is individually fixed dynamically independent of each other at fixed values of $\beta_{b}$ 's.

(*) Cf. Eq. (3.1) 
A Method of Statistical Mechanical Treatment of Equilibrium and Chernical Reactions.

A simple example of such an assembly is that consisting of two gas ous perts $A$ and $K$ sealed in a cylinder and separated by a frictionless piston left free to move; the energy state of the either gas may be taken is $\mathrm{d} t \times \mathrm{rmined}$ by the volume of $A$, which is the only external paramet $x$ of the latter propar, and no force conjugated to the parameter is exert d by the extornal world.

We have thus in thass asmbly $A+K$ the special case of that dealt with in ti foregoing sctions, for which, of course, all definitions and cons quent thooretical develoyments remain valid. The same is true with the s.s ${ }^{\circ}$ mbly $A$ alon provided that $\beta_{b}$ 's are arrested. The latter assembly will be denot d by $A_{3}$.

We may thus $d$ fin' $\lambda$ 's eithar with the assembly $A+K$ or with $A_{8}$ sp cifying the assambly $C$ of Eq. (3.5) to be either $A+K$ or $A_{A}$. Former on $s$ will be celled $\lambda$ 's of assembly $A$ of variable external paramet rs o: simply thos of $A+K$ and lettrs those of $A_{\mathfrak{p}}$.

In ti following s ction we will first show the identity of the two series of $\lambda$ 's of the alt rnotive definitions $(\$ 10)$ next transform $\lambda$ 's of $A+K$ with special refenes to the intrested assembly $A(\S 11)$ and finally put tis resulting expressions in comparison with those for $\lambda$ 's of $A_{\mathrm{p}}$ i to to tomodynamicel t rminology $(\S 12)$.

$\$ 10$. Identity of $\lambda$ 's of the Assmbly $A+K$ and Those of $A_{\sharp}$.

$\log \lambda$ is express a d acording to Eq. (4.3) in the form,

$$
\left.\log \lambda=J_{\lambda} \log \mathfrak{Q}(A+K)=\left(\nu_{\lambda} \log \mathfrak{Q}(A+K)\right)_{\beta}+\sum_{\beta} \frac{\partial \log \mathfrak{O}(A+K)}{\partial \beta_{b}}\right\lrcorner_{\lambda} \beta_{b}
$$

wher suffi $\beta$ signifi s th condition of fixed $\beta_{b}$ 's and $\Delta_{\lambda} \beta_{b}$ the increments of $\beta_{b}$ 'nd $\mathrm{r}$ the speif $\mathrm{d}$ condition of $A+K$. The differential coefficient $\frac{\partial \log \cap(A+K)}{\partial A_{b}}$ is however according to Eq. (7.2) the force conjugated to $\beta$ multplied by $1 / i T$, which vanishes according to (ii), $\$ 9$, i.e.,

$$
\frac{\partial \operatorname{lor} \mathfrak{Q}(A+K)}{\partial \beta_{b}}=0
$$

and $h$ ne tio second tarn on the third member of Eq. (10.1) vanishes.

Th first term $\left(J_{\lambda} \log _{0} \mathfrak{Q}(A+K)\right)_{\mathrm{s}}$ is expressed in the form,

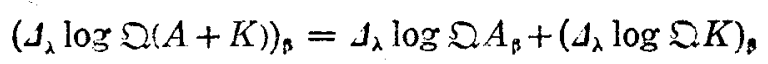


where

$$
\left(\Delta_{\lambda} \log \mathfrak{Q} K\right)_{\beta}=0
$$

since $A$ is only subjected to the operation.

We have by Eqs. (10.1), (10.2), (10.3) and (10.4) that,

$$
\log \lambda=\Delta_{\lambda} \log \mathfrak{Q}(A+K)=\Delta_{\lambda} \log \mathfrak{Q} A_{\beta}
$$

which states the enunciated identity.

$\$ 11$. Expression of $A$ and Derived Quantities with Special Reference to $A$.

We begin with the expression for $-k T \log \mathfrak{Q}(A+K)$ with special reference to the assembly $A$. According to (iii), $\$ 9, \mathfrak{Q}(A+K)$ is factored in the form,

$$
\mathfrak{Q}(A+K)=\mathfrak{\Omega} A \cdot \mathfrak{D} K
$$

and $\Omega K$ in turn expressed according to $\mathrm{Eq} .(7.1)$ as that,

$$
\log \square K=\frac{1}{k T} \sum_{b} \int_{B_{b}(0)}^{\beta_{b}} I_{\beta_{b}}^{K} d \beta_{b}+\log \supseteq K_{\beta(0)}
$$

where $J_{\beta_{b}}^{K}$ is the average force of the assembly $K$ conjugated to $\beta_{b}$ and $K_{i(n)}$ the assembly $K$ fixed by a particular set of values $\beta_{b}(0)$ 's of $\beta_{b}$ 's.

We have, on the other hand, according to Eqs. (10.2), (11.1) and (7.2),

$$
H_{\mathrm{B}, b}^{A}+H_{i b}^{K}=0
$$

where $I_{s, b}^{A}$ is the force of the assembly $A$ conjugated with $\beta_{b}$. Substituting $\Pi_{* h}^{K}$ from Eq. (11.3) into Eq. (11.2) we have,

$$
\log Q \mathcal{Q} K=-\frac{1}{k T} \sum_{b} \int_{\beta_{b}(0)}^{\beta_{n}} I_{\beta_{b}}^{A} d \beta_{b}+\log \mathfrak{Q} K_{\beta(0)}
$$

and substituting the latter in turn into Iq. (11.1),

$$
\log \mathfrak{Q}(A+K)=\log \mathfrak{D A}-\frac{1}{k T} \sum \int_{\beta_{b}(0)}^{\beta_{b}} \Pi_{\beta_{b}}^{A} d \beta_{b}+\log \mathfrak{Q} K_{\beta_{(0)}}
$$

Eq. (11.5) expresses $\log \mathfrak{Q}(A+K)$ in term of variables appropriate to $A, \log \mathfrak{O} K_{i(0)}$ being thereby a constant.

Eqs. (11.5) and (3.5) give now according to Eq. (4.3), that 
A Method of Statistical Mechanical Treatment of Equilibrium and Chemical Reactions.

$$
\log \lambda=J_{\lambda} \log \Omega A-\frac{1}{k T} \sum_{b} \int_{\beta_{b}(0)}^{\beta_{b}} \Delta_{\lambda} I_{\beta_{b}}^{A} d \beta_{b}-\frac{1}{k T} \sum_{i} I_{\beta_{b}}^{A} \Delta_{\lambda} \beta_{b}
$$

where $A_{\lambda} I_{b,}^{A}$ is the increment of the force $I I_{\beta,}^{A}$, due to the operation associated with $\lambda$.

The temperature variation of $\mathfrak{Q}(A+K)$ and of $\lambda$ is now derived begining again with that of $\Omega(A+K)$. We have according to Eqs. (10.2) and (11.1) that,

$$
\begin{aligned}
\frac{\partial \log \mathfrak{Q}(A+K)}{\partial T} & =\left(\frac{\partial \log \mathfrak{Q}(A+K)}{\partial T}\right)_{\beta}+\sum_{\beta} \frac{\partial \log \Omega(A+K)}{\partial \beta_{b}} \frac{\partial \beta_{b}}{\partial T} \\
& =\left(\frac{\partial \log \Omega A}{\partial T}\right)_{\beta}+\left(\frac{\partial \log \supseteq K}{\partial T}\right)_{B}
\end{aligned}
$$

or expressing $\left(\frac{\partial \log \mathfrak{Q} K}{\partial T}\right)$, by Eq. (11.4) that,

$$
\begin{gathered}
k T^{2} \frac{\partial \log Q(A+K)}{\partial T}=k T^{2}\left(\frac{\partial \log \Omega A}{\partial T}\right)_{\beta}+\sum_{b} \int_{\beta_{b}(0)}^{\beta_{b}} I I_{\beta_{h}}^{A} d \beta_{b} \\
\quad-T \sum_{b} \int_{b_{b}(0)}^{\beta_{b}} \frac{\partial I_{r_{b}}^{A}}{\partial T} d \beta_{b}+k T^{2} \frac{\partial \log \Omega K_{B(0)}}{\partial T}
\end{gathered}
$$

where the partial differential coefficient without suffix denotes those under the specified condition of $A+K$ in distinction from those with suffix $\beta$ at fixed $\beta_{b}$ 's.

The temperature variation of $\lambda$ is expressed according to $\$ 4$, Eqg. (3.5) and (11.8) as that,

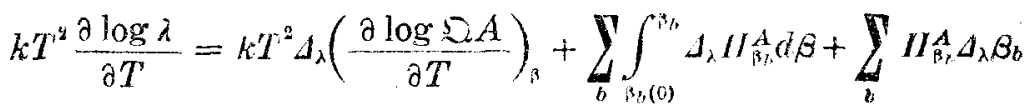

$$
\begin{aligned}
& -T \sum_{b} \int_{\beta_{b}(0)}^{\beta_{b}^{\prime}} A_{\lambda} \frac{\partial I I_{\beta_{b}}^{A}}{\partial T^{\prime}} d \beta-T \sum_{b} \frac{\partial I I_{i, b}^{A}}{\partial T^{\prime}} J_{i, \beta_{b}}
\end{aligned}
$$

Eq. (11.9) is of course directly obtained by the differentiation of Eq. (11.6).

The temperature variation of $\lambda$ denined with respect to assembly $A_{\mathfrak{\beta}}{ }^{(*)}$ is expressed according to Eqs. (3.5), (11.1) and (10.4) as that,

(*) Cf. $\$ 9$. 


$$
\begin{aligned}
& \text { Journal of the Research Institute for Cataysis } \\
& k T^{2}\left(\frac{\partial \log \lambda}{\partial T}\right)_{\beta}=k T^{2} d_{\lambda}\left(\frac{\partial \log \Omega A}{\partial T}\right)_{\beta}
\end{aligned}
$$

Comparing Eq. (11.9) with (11.10), we see that whereas $\lambda$ 's of the two alternative definitions are identical as shown in $\$ 10$, their temperature variation are different in general.

\$12. Thermodynamical Form of $\lambda$ 's and Associated Quantities for the Assembly of Variable External Parameters.

We first write down Helmholtz free energy and the internal energy respectively according to Eq. (8.1) and to Eq. (8.2) of the assemblies, $A+K, A$ and $K_{8(0)}$ with appropriate subscripts, i.e.,

$$
\begin{aligned}
& F=-k T \log \mathfrak{D}(A+K) \\
& F_{A}=-k T \log \mathfrak{D A} \\
& F_{K, \beta(0)}=-k T \cdot \log \mathfrak{D} \boldsymbol{K}_{\beta(0)} \\
& U=k T^{2} \frac{\partial \log \mathfrak{\Omega}(A+K)}{\partial T} \\
& U_{A}=k T^{2}\left(\frac{\partial \log \mathfrak{Q} A}{\partial T}\right)_{\beta}^{(*)} \\
& * \quad U_{K_{1} \cap(0)}=k T^{2} \frac{\partial \log \mathfrak{D} K_{\bullet(0)}}{\partial T}
\end{aligned}
$$

Thermodynamical forms are obtained by simply rewriting expressions obtained in the previous section in terms of F's and $U$ 's as follows,

$$
\begin{gathered}
F=F_{A}+\sum_{b} \int_{\beta(0)}^{\beta_{b}} I_{\beta_{b}}^{A} d \beta_{b}+F_{K \cdot \beta(0)} \\
-k T \log \lambda=\Delta_{\lambda} F_{A}+\sum_{b} I_{\beta_{b}}^{A} \Delta_{\lambda} \beta_{b}+\sum_{b} \int_{\beta_{b}(0)}^{\beta_{b}} \Delta_{\lambda} I I_{\beta_{b}}^{A} d \beta_{b}
\end{gathered}
$$

(*) External parameters of $A$ proper must be fixed at the partial differentiation for obtaining the internal energy of $A$ with regard to the implied condition of the general expression Eq. (8.2). 
A Method of Statistical Mechaniral Treatment of Equilibrium and Chemical Reactione.

(11.8)

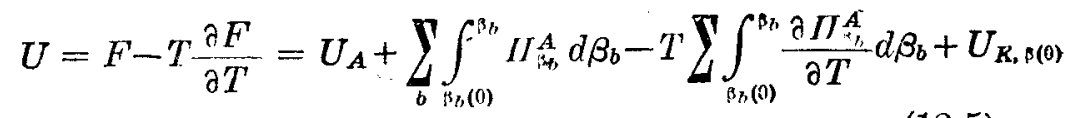

(11.9)

$$
\begin{aligned}
k T^{2} \frac{\partial \log \lambda}{\partial T}= & \lrcorner_{\lambda} U_{A}+\sum_{b} I_{\beta_{b}}^{A} d_{\lambda} \beta_{b}+\sum_{b} \int_{b(0)}^{\beta_{b}} \Delta_{\lambda} I_{b_{b}}^{A} d \beta_{b} \\
& -T \sum_{b} \frac{\partial I I_{\beta_{b}}^{A}}{\partial T} J_{\lambda} \beta_{b}-T \sum_{b} \int_{\beta_{b}(0)}^{\beta_{b}} \Delta_{\lambda} \frac{\partial \Pi_{B_{b}}^{A}}{\partial T} d \beta_{b}
\end{aligned}
$$

\$13. Thermodynamical Form of $\lambda$ 's and Derived Quantities for the Assembly $A_{P}$.

Thermodynamical form of $\quad-k T \log \mathfrak{Q}(A+K), \quad-k T \log \lambda$; $k T^{2} \frac{\partial \log \mathfrak{Q}(A+K)}{\partial T}$ and $k T: \frac{\partial \log \lambda}{\partial T}$ derived in the previous section with special reference to the assembly $A$ correspond in general to no readymade thermodynamical functions except when $\lambda \equiv p^{\circ}$ and $A \equiv A_{P}$ or when only external parameter of $A$ is the volume $V_{A}$ and the conjugated average force the pressure $\mathrm{P}_{A}$ is kept constant independent of $V_{A}$ and $T$.

Thermodynamical form in the previous section are developed below with this particular case that $\beta \equiv V_{A}, I^{A} \equiv P_{A}=$ constant, and $\lambda \equiv p^{\delta}$ or $-R T \log \lambda=\mu^{8}$, first rewriting the expressions there as follows;

$$
\begin{aligned}
& \mathrm{P}_{A}=-\left(\frac{\partial F_{A}}{\partial V_{A}}\right)_{T} \\
& F=F_{A}+P_{A} V_{A}+F_{K, V_{A}=0} \\
& \mu^{\delta}=F_{A}^{\delta}+P_{A} V_{A}^{\delta} \\
& U=F-T \frac{\partial F}{\partial T}=U_{A}+P_{A} V_{A}+U_{K, V_{A}=0} \\
& \mu^{\delta}-T \frac{\partial \mu^{\delta}}{\partial T}=\bar{U}_{A}^{\delta}+P_{A} \bar{V}_{A}^{\delta}
\end{aligned}
$$

, $F_{K, v_{A}=0}$ and $U_{K, v_{A}=0}$ give respectively particular values of $E_{K,:(0)}$ and $U_{K, \beta(0)}$ at $\beta(0) \equiv V_{A}(0)=0$, and,

$$
\bar{F}_{A}^{\delta}=\Delta_{\mathfrak{p}} F_{A}, \quad \bar{U}_{A}^{\delta}=\Delta_{p} U_{A}, \quad \bar{V}_{A}^{\delta}=\Delta_{p} V_{A} \quad(13.6 . \mathrm{F}),(13.6 . \mathrm{U}),(13.6 . \mathrm{V})
$$


are pertin molal quantities at the particular condition of $A_{P}$ i.e. at the consta it pressure.

We see the variable part $H_{A}+P_{A} V_{A}$ of $F$ in Eq. (13.2) correspond to Gibbs iree energy $Z_{A}$, that $U_{A}+P_{A} V_{A}$ of $U$ in $\mathrm{Eq}$. (13.4) to the othalp $X_{A},{ }_{A}^{B}+P_{A} \bar{V}_{A}^{i}$ of Eq. (13.3) to the partial molal quantity $\bar{Z}_{A}^{\delta}$ of $Z_{A}$ ard $\bar{U}_{A}^{\delta}+P_{A} \bar{V}_{A}^{\delta}$ of Eq. (13.5) to the partial molal enthalpy $\bar{X}_{A}^{\delta}$ of $X_{A}, i, \therefore$,

$$
\begin{aligned}
& Z_{A}=F_{A}+P_{A} V_{A} \\
& X_{A}=U_{A}+P_{A} V_{A} \\
& \bar{Z}_{A}^{\delta}=\overline{\bar{T}}_{A}^{o}+P_{A} \bar{V}_{A}^{\delta}
\end{aligned}
$$

and

$$
\bar{X}_{A}^{\hat{\delta}}=\bar{U}_{A}^{\delta}+P_{A} \bar{V}_{A}^{\delta}
$$

In term of these "ready-made" thermodynamical functions previous equations are respectively rewritten as follows:

$$
\begin{aligned}
& \left(\frac{\partial Z_{A}}{\partial P_{A}}\right)_{T}=V_{A}^{(*)} \\
& \mu^{\delta}=Z_{A}^{\delta} \\
& Z_{A}-T \frac{\partial Z_{A}}{\partial T}=X_{A}^{(* *)} \\
& \mu^{\delta}-T \frac{\partial \mu^{\delta}}{\partial Z^{\gamma}}=\bar{X}_{A}^{\delta}
\end{aligned}
$$

Theororating Wos. (13.11) and (13.12), we have further,

(*) Exrressing $\left(\frac{\partial T_{A}}{\partial P_{A}}\right)_{T}$ by differentiation of Eq. (12.7) in the form

$$
\left(\frac{\partial Z_{A}}{\partial P_{A}}\right)_{T}=\left(\frac{\partial F_{A}}{\partial V_{A}}\right)_{T}\left(\frac{\partial V_{A}}{\partial P_{A}}\right)_{T}+P_{A}\left(\frac{\partial V_{A}}{\partial P_{A}}\right)_{T}+V_{A}
$$

we have Eq. (13.11) from Eq. (13.1).

(**) Substituting $F$ into Eq. (13.4) from (13.2) and observing Eq. (13.7), (15.8) and the relation

$$
F_{K}, V_{A}=0-T \frac{\partial F_{K} \cdot V_{A} 0}{\partial T}=U_{K}, V_{A} \cdot 0
$$

valid as a special case of Eq. (8.3), we have the above relation. 
A Method of Statistical Mechanical Treatment of Equilibrium and Chemical Réactions.

$$
\left(\begin{array}{c}
\partial \mu^{\delta} \\
\partial P_{A}
\end{array}\right)_{T}=V_{A}^{\delta}
$$

These relations are obtained in alternative forms by introducing the entropy $S_{A}$ of the assambly $A$ proper, according to $\mathrm{Eq}$. (8.8), i. e.,

$$
S_{A}=-\left(\frac{\partial F_{A}}{\partial T}\right)_{V_{A}}^{(*)}
$$

We have thus from $\mathrm{Eq} .(13.7)$,

$$
S_{A}=-{\frac{\partial Z_{A}}{\partial T * *)}}^{(* *)}
$$

from Eq. (13.13),

$$
Z_{A}+T S_{A}=X_{A}
$$

from (13.17) and (13.12)

$$
\bar{S}_{A}^{\delta}=-\frac{\partial \mu^{\delta}}{\partial T}
$$

and from (13.12) and (13.18)

$$
\mu^{\delta}+T \bar{S}_{A}^{s}=\bar{Y}_{A}^{s}
$$

where partial differential coefficients without suffix and the partial molal quantity $\bar{S}_{A}^{\delta}$ are all referred to the specified condition of $A_{P}$ i.e. to the constant pressure.

We rewite thess relations for partial molal quantities of $A$ for the sake of later applications dropping the subseript $A$ signifying instead explicitly the specified condition by suffix $P$.

$$
\mu^{\hat{n}}=Z_{P}^{i}
$$

(*) The external parameter of $A$ proper i.e. the volume must be kept constant at the partial differentiation with regard to the implied condition of the general exprossion Eq. (8.8).

(*) According to the identity, $\left(\frac{\partial F_{A}}{\partial T}\right)_{P_{A}}=\left(\frac{\partial F_{A}}{\partial T}\right)_{V_{A}}+\left(\frac{\partial F_{A}}{\partial V_{A}}\right)_{T}\left(\frac{\partial V_{A}}{\partial T}\right)_{P_{A}}$ and Eqs. (13.16), (13.1) and (13.7) we have above relation, where $\left(\frac{\theta Z_{A}}{\partial T}\right)_{P_{A}}$ is expressed without suffix. 


$$
\begin{aligned}
& \left(\frac{\partial \mu^{\delta}}{\partial T}\right)_{P}=-\bar{S}_{P}^{\delta} \\
& \mu^{\delta}=\bar{X}_{P}^{\delta}-T \bar{S}_{P}^{\delta}
\end{aligned}
$$

Corresponding relations for $A_{V}$ of the assembly $A$, whose only external parameter $V$ is fixed, may be written down as follows as the special case of those given in $\$ 8$, signifying explicitly the constancy of the volume, i.e.,

and

$$
\begin{aligned}
& \mu^{\delta}=\bar{F}_{A}^{\delta} \\
& \left(\frac{\partial \mu^{\delta}}{\partial T}\right)_{V}=-\bar{S}_{V}^{\delta}
\end{aligned}
$$

The relation between two groups of partial molal quantities appearing respectively in Eqs. (13.21) and (13.22) is developed as below.

It is immediately transposed from Eqs. (13.9), (13.10) and (13.15) that,

$$
\begin{aligned}
& \bar{Z}_{P}^{\delta}=\bar{F}_{P}^{\delta}+P \bar{V}_{P}^{\delta} \\
& \bar{X}_{P}^{\delta}=\bar{C}_{P}^{\delta}+P \bar{V}_{P}^{\delta} \\
& \left(\frac{\partial \mu^{\delta}}{\partial P}\right)_{T}=\bar{V}_{P}^{\delta} .
\end{aligned}
$$

It follows on the other hand from the identity of $p^{\delta}$ or $\mu^{\delta}$ of the assembly $A_{P}$ with that of $A_{V}$ as verified in $\$ 10$ and according to Eqs. (13.21.E) and (13.22.E) that,

$$
\mu^{\delta}=\bar{X}_{P}^{\delta}-T \bar{S}_{P}^{\delta}=\bar{U}_{V}^{\delta}-T \bar{S}_{V}^{\delta}
$$

We derive from Eqs. (13.21.S) that,

$$
\bar{S}_{P}^{\delta}=-\left(\frac{\partial \mu^{\delta}}{\partial T}\right)_{P}=-\left(\frac{\partial \mu^{\delta}}{\partial T}\right)_{V}+\left(\frac{\partial \mu^{\delta}}{\partial P}\right)_{T}\left(\frac{\partial P}{\partial T}\right)_{V}
$$

or according to Eqs. (13.23.V), (13.22.S) and to the relation

$$
\left(\frac{\partial P}{\partial T}\right)_{V}=-\frac{1}{V}\left(\frac{\partial V}{\partial T}\right)_{P} / \frac{1}{V}\left(\frac{\partial V}{\partial P}\right)_{T}
$$

that, 
A Method of Statiotical Mechanical Treatment of Eguilibrium and Chemical Reactions.

$$
\bar{S}_{p}^{\delta}=\bar{S}_{V}^{\delta}+\frac{\alpha}{\beta} \bar{V}_{P}^{\delta}
$$

where

$$
\alpha=\frac{1}{V}\left(\frac{\partial V}{\partial T}\right)_{P} \quad \text { and } \quad \beta=-\frac{1}{V}\left(\frac{\partial V}{\partial P}\right)_{T}
$$

are the expansion coefficient and the compressibility respectivley.

From Eqs. (13.23.S), (13.23. $\mu$ ) and (13.23.X) we have,

$$
\bar{X}_{P}^{\delta}=\bar{U}_{V}^{\delta}+T \frac{\alpha}{\beta} \bar{V}_{P}^{\delta}
$$

and

$$
\bar{U}_{P}^{\delta}=\bar{U}_{V}^{\delta}+\left(T \frac{a}{\beta}-P\right) \bar{V}_{P}^{\delta}
$$

Finally we derive from Eq. (13.23. $\mu),(13.21 . S)$ and (13.23.V) that,

$$
\left(\frac{\partial \bar{X}_{P}^{o}}{\partial P}\right)_{T}=\bar{V}_{P}^{\delta}-T\left(\frac{\partial \bar{V}_{P}^{\delta}}{\partial T}\right)_{P}
$$

\section{\$14. Statistical Thermodynamics of Homogeneous Fluid.}

We call such an assembly or such a spacial part of an assembly a homogeneous fluid that only external parameter is volume and $Q^{b}$ of every constituent molecule is respectively constant all over the space, depending only on temperature, pressure and on the composition but not on its absolute magnitude.

A homogeneous fluid is called ideal solution with respect to certain components if $Q^{\delta}$ of the latters are independent of any concentration of these components. The latters are called ideal components, the homogeneons fluid ideal with respect to the components and the homogeneous fluid consisting of remaining components the solvent. We may expect a homogeneous fluid is ideal with respect to a component which is dilute enough so that the work $-k T \log q_{o}^{\delta}$ and hence $Q^{\delta}$ of every constituent is constant independent of the concentration of the dilute component.

A homogeneous fluid is called a perfect gas or simply a gas if the latter is ideal with respect to every component.

Followings are deduced from the above definition.

Let an ideal solution $A$ with respect to a component $\delta_{0}$ be separated from its solvent $B$ consisting of $\delta_{1}, \cdots, \delta_{s}$ by a semipermeable membrane 
which is impermeable only to $\delta_{0}$. The whole assembly is considered as in equilibrium at a constant volume, and $B$ big enough so that the shift of the membrane practically makes no difference in its pressure nor in composition.

Since $Q^{s}$ 's and hence $\mu^{\delta}$ 's according to Eqs. (5.5) and (8.12) depend only on the pressure and composition but not on the absolute magnitude of the fluid at constant temperature, Gibbs' free energy $Z$ of a homogeneous fluid is expressed according to $\mathrm{Eq} .(13.21 . \mu)$ in the form,

$$
Z=\sum_{i} n^{\delta_{i}} \mu^{\delta_{i}}
$$

and hence $F$ according to $\mathrm{Eq}$. (13.7),

$$
F=\sum n^{\delta} \mu^{\delta}{ }^{\delta}-P V
$$

where $n^{\delta_{i}}$ is the number of mols of $\delta_{i}$.

The $F$ of the part $A$ or $B$ is now respectively expressed as that,

$$
F_{A}=n^{\delta_{0}} \mu^{\delta_{0}}+\sum_{i=1}^{i=g} n_{A}^{\delta_{i}} \mu_{A}^{\delta_{i}}-P_{A} V_{A} \quad \text { or } \quad E_{B}=\sum_{i=1}^{i-s} n_{B}^{\delta_{i}} \mu_{B}^{\delta_{i}}-P_{B} V_{B}
$$

Because of the assumed equilibrium we have, however, according to Eqs. (6.1) and (8.12) that,

$$
\mu_{\Lambda}^{\delta_{i}}=\mu_{B}^{\delta_{i}}=\mu^{\delta_{i}}=\mathrm{constant}, \quad i=1, \cdots, \xi
$$

Denoting the sum $n_{A}^{\delta_{i}}+n_{\beta_{i}}^{\delta_{i}}$ by $n^{\delta_{i}}$ we have for $F$ of the whole assembly that,

$$
\vec{F}=F_{A}+F_{B}=n n^{\delta_{0}} l^{\delta_{0}}+\sum_{i} n^{\delta_{i}} \mu^{\delta_{i}}-P_{A} V_{A}-P_{B} V_{B}
$$

Differentiating $F$ with respect to $V_{A}$ we obtain the average force $I^{\delta_{0}}=P_{A}-P_{B}$ conjugated with $V_{A}$ or the osmotic pressure,

$$
\begin{gathered}
P_{A}-P_{B}=-\left(\frac{\partial F}{\partial V_{A}}\right)_{T}=-n^{\varepsilon_{0}}\left(\frac{\partial \mu^{\delta_{0}}}{\partial V_{A}}\right)_{T}+P_{A}-P_{B}+V_{A}\left(\frac{\partial P_{A}}{\partial V_{A}}\right)_{T} \\
\text { or } \quad V_{A}\left(\frac{\partial P_{A}}{\partial V_{A}}\right)_{T}=n^{\delta_{0}}\left(\frac{\partial \mu^{\delta_{0}}}{\partial V_{A}}\right)_{T}
\end{gathered}
$$

with regard to the relation that $V_{A}+V_{B}=$ constant.

According to Eqs. (5.5) and (8.12) we have that, 
A Method of Statistical Mechanical Treatment of Euilibrium and Chemical Reactions.

$$
\mu^{\delta}=\mu_{1}^{\delta}+R T \log N^{\delta} \quad \text { where } \quad \mu_{1}^{\delta}=-R T \log Q^{\delta} \quad(14.5),(14.6)
$$

and bence noting $N^{\varepsilon_{0}}=n^{\delta_{0}} / V_{A}$ that,

$$
\left(\frac{\partial \mu^{\gamma_{0}}}{\partial V_{A}}\right)_{T}=\left(\frac{\partial \mu_{1}^{\hat{f}_{0}}}{\partial P_{A}}\right)_{T}\left(\frac{\partial P_{A}}{\partial V_{\mathrm{A}}}\right)_{T}-\frac{R T}{V_{A}}
$$

inasmuch as $Q^{\delta}$ of the ideal component is the function only of pressure at a constant temperature. $\left(\frac{\partial \mu_{1}^{\gamma} 0}{\partial P_{A}}\right)_{T}$ is obtained from Eq. (14.5) as that,

$$
\left(\frac{\partial \mu_{10}^{\delta_{0}}}{\partial P_{A}}\right)_{T}=\bar{V}_{P}^{\delta_{0}}=\left(\frac{\partial \mu_{1}^{\beta_{0}}}{\partial P_{A}}\right)_{T}+R T \beta_{A}
$$

where

$$
\beta_{A}=-\left(\frac{\partial \log V_{A}}{\partial P_{A}}\right)_{T}
$$

is the compressibility.

By Eqs. (14.4), (14.7) and (14.8), we have that,

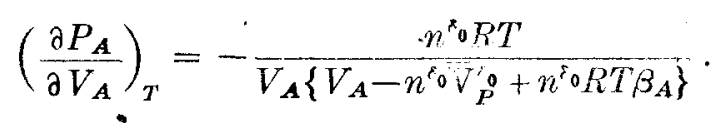

or by integration, remembering that $I^{0_{0}}=P_{A}-F_{B}$,

$$
I I^{\delta_{0}}=n^{\delta_{0}} R T \int_{V_{A}}^{\infty} \frac{d V_{A}}{V_{A}\left\{V_{A}-n^{\delta_{0}} \bar{V}_{P}^{\rho_{0}}+n^{i 0} R T \beta_{A}\right\}}
$$

provided that $P_{A}=P_{B}$ at $V_{A}=\infty$.

If $n^{*} \cdot R T \beta_{A}$ is negligible compared with $V_{A}-n^{\varepsilon_{0}} \bar{V}_{P}^{\hat{\theta}_{0}}$ and $\bar{V}_{P}^{\gamma_{0}}$ is constant independent of $V_{A}$, which might be the case for a colloidal solution, $n_{0}^{\delta_{0}} \bar{V}_{P}^{\delta_{n}}$ being the total displacement volume of the colloidal particles, Eq. (14.9) assumes the form with regard to the relation $N^{\varepsilon_{0}}=n^{i} / V$ that,

$$
I^{\delta_{0}}=-\frac{R T}{\bar{V}_{P}^{\delta_{0}^{-}}} \log \left(1-N^{\delta_{0}} \bar{V}_{P}^{\delta_{0}}\right)
$$

Eq. (14.10) might be useful for the determination of the molecular weight of the colloidal particles by analysing the measurement of $I^{\delta_{0}}$ at different concentrations, whereby the displacement volume $n^{\delta} \bar{V}_{P}^{\delta_{0}}$ is simultaneously determined. 
If $n^{\delta_{0}} \bar{V}_{P}^{\delta_{0}}-n^{\delta}{ }_{0} R T \beta_{A}$ is negligibly small compared with $V_{A}$ we have from Eq. (14.9) that,

$$
I^{\delta_{0}}=n^{\delta_{0}} \frac{R T}{V}
$$

If ideal components $\delta_{0}, \cdots, \delta_{t}$ are confined together in the space $A$, we have by similar reasoning instead of Eq. (14.9), that,

$$
I I=\sum_{i=0}^{i=t} n^{\delta_{i}} R T \int_{V_{A}}^{\infty} \frac{d V_{A}}{V_{A}\left\{V_{A}-\sum_{i=0}^{i-t} n^{\delta_{i}}\left(\bar{V}_{\boldsymbol{P}}^{\delta_{i}}-R T \beta_{A}\right)\right\}}
$$

and instead of Eq. (14.11), if $\sum_{i=0}^{i-t} n^{\delta_{i}}\left(\bar{V}_{P}^{\delta_{i}}-R T \beta_{A}\right)$ is negligibly small compared with $V_{A}$, that

$$
I I=\sum_{i=0}^{i-t} n^{\delta_{i}} \frac{R T}{V_{A}}
$$

In the further special case of a gas, when the assembly is ideal with respect to every component we have according to our definition that, $\left(\frac{\partial t_{1}^{8} 0}{\partial P}\right)_{T}=0$, since the pressure varies nothing but the concentration of ideal components. We have hence according to Eq. (14.8), that,

$$
R T \beta-\bar{V}_{P}^{\delta_{i}}=0, \quad i=1, \cdots, s
$$

or taking the fact thereby that $P_{B}=0$ or $I I=P_{A}$ into account and dropping subscript $A$ that,

$$
P V=\sum_{i=0}^{i-s} n^{\delta_{i}} R T
$$

i.e. the ideal gas law. It may be noted that Eq. (14.14) is conversely satisfied by Eq. (14.15) inasmuch as $\beta=1 / P$ and $\bar{V}_{p}^{\delta_{i}}=R T / P$ according to the latter.

For a general homogeneous fluid we define $\bar{F}_{V, 1}^{\delta}, " \bar{F}_{P, 1}^{s}$ and $\bar{Z}_{P, 1}^{s}$ as that,

$$
\begin{gathered}
\bar{F}_{V, 1}^{\delta}=\bar{F}_{V}^{\delta}-R T \log N^{\delta} \\
\bar{F}_{P .1}^{\delta}=\bar{F}_{P}^{\delta}-R T \log N^{\delta} \\
-30-
\end{gathered}
$$


A. Method of Statistical Mechanical Treatment of Equilibrium and Chemical Reactions.

$$
\bar{Z}_{P, 1}^{\delta}=\bar{Z}_{P}^{\delta}-R T \log N^{\delta}
$$

It follows immediately from Eqs. (14.16), (14.5), (13.22. $\mu),(13.21 . \mu)$ and (13.23.Z) that,

$$
\mu_{1}^{\delta}=\bar{F}_{V, 1}^{\delta}=\ddot{F}_{P, 1}^{\delta}+P \bar{V}_{P}^{\delta}=\bar{Z}_{P_{1}^{1}}^{\delta}
$$

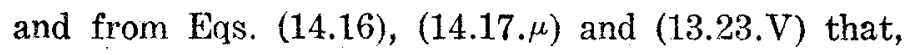

$$
\left(\frac{\partial \bar{F}_{V, 1}^{\delta}}{\partial P}\right)_{T}=\left(\frac{\partial \bar{Z}_{P, 1}^{\delta}}{\partial P}\right)_{T}=\bar{V}_{P}^{\delta}-R T \beta
$$

remembering that,

$$
N^{\delta}=n^{\delta} / V
$$

$\bar{S}_{V, 1}^{\delta}$ and $\bar{S}_{P, 1}^{\delta}$ are defined as follows;

$$
\begin{aligned}
& \bar{S}_{V, 1}^{\delta}=\bar{S}_{V}^{\delta}+R \log N^{\delta} \\
& \bar{S}_{P .1}^{\delta}=\bar{S}_{P}^{\delta}+R \log N^{\delta}
\end{aligned}
$$

We have on the other hand differentiating $\mu^{\delta}$ of $\mathrm{Eq}$. (14.5) with respect to $T$ respectively with constant $V$ or $P$ that,

$$
\begin{aligned}
& \left(\frac{\partial \mu^{\delta}}{\partial T^{\delta}}\right)_{V}=\left(\frac{\partial \mu_{1}^{\delta}}{\partial T}\right)_{V}+R \log N^{\delta} \\
& \left(\frac{\partial \mu^{\delta}}{\partial T}\right)_{P}=\left(\frac{\partial \mu_{1}^{\delta}}{\partial T}\right)_{P}-R T \alpha+R \log N^{\delta}
\end{aligned}
$$

It follows from Eqs. (14.18), (13.22.S), (13.21.S) and (14.19) that,

$$
\bar{S}_{V, 1}^{\delta}=-\left(\frac{\partial \mu_{1}^{\delta}}{\partial T}\right)_{V}, \quad \bar{S}_{P, 1}^{\delta}=-\left(\frac{\partial \mu_{i}^{0}}{\partial T}\right)_{P}+R T a
$$

- and from Eqs. (14.18) and (13.23.S) that,

$$
\bar{S}_{P, 1}^{\delta}=\bar{S}_{V, 1}^{\delta}+\frac{a}{\beta} \bar{V}_{P}^{\delta}
$$

$\bar{U}_{V, 1}^{\delta}, \bar{U}_{P, 1}^{\delta}$ and $\bar{X}_{P, 1}^{\delta}$ are now defined as that,

$$
\begin{gathered}
\bar{U}_{V, 1}^{\delta}=\bar{F}_{V, 1}^{\delta}+T \bar{S}_{V, 1}^{\delta} \\
-31-
\end{gathered}
$$




$$
\begin{aligned}
& \bar{U}_{P, 1}^{\delta}=\bar{X}_{P, 1}^{\delta}-P \bar{V}_{P}^{\delta} \\
& \bar{X}_{P, 1}^{\delta}=\bar{Z}_{P, 1}^{\delta}+T \bar{S}_{P, 1}^{\delta}
\end{aligned}
$$

along with the expressions respectively for $\bar{U}_{V}^{\delta}, \bar{U}_{P}^{\delta}$ and $\bar{X}_{P}^{\delta}$ as derived from Eqs. (13.21), (13.22) and (13.23) i.e.,

$$
\begin{aligned}
& \bar{U}_{V}^{\delta}=\bar{F}_{V}^{\delta}+T \bar{S}_{V}^{\delta} \\
& \bar{U}_{P}^{\delta}=\bar{X}_{P}^{\delta}-P \bar{V}_{P}^{\delta} \\
& \bar{X}_{P}^{\delta}=\bar{Z}_{P}^{\delta}+T \bar{S}_{P}^{\delta}
\end{aligned}
$$

If follows from Eqs. (14.16), (14.18), (14.21) and (14.22) that,

$$
\bar{U}_{V, 1}^{\delta}=\bar{U}_{V}^{\delta}, \bar{U}_{P, 1}^{\delta}=\bar{U}_{P}^{\delta}, \bar{X}_{P, 1}^{\delta}=\bar{X}_{P}^{\delta} \quad\left(14.23 . U_{V}\right),\left(14.23 . U_{P}\right),\left(14.23 . \mathrm{X}_{P}\right)
$$

and hence from Eqs. (14.23), (13.24) and (13.25) that,

$$
\begin{aligned}
& \bar{X}_{P, 1}^{\delta}=\bar{U}_{V, 1}^{\delta}+T \frac{a}{\beta} \bar{V}_{P}^{\delta} \\
& \bar{U}_{P, 1}^{\delta}=\bar{U}_{V, 1}^{\delta}+\left(T \frac{a}{\beta}-P\right) \bar{V}_{P}^{\delta} \\
& \left(\frac{\partial \bar{X}_{P, 1}^{\delta}}{\partial P}\right)_{T}=\bar{V}_{P}^{\delta}-T\left(\frac{\partial \bar{V}_{P}^{\delta}}{\partial T}\right)_{P}
\end{aligned}
$$

In the case of ideal solution for which $\mu_{1}^{8}$ is constant at constant $T$ and $P$ the above quantities with suffix 1 are all similarly constant independent of $N^{\delta}$ provided that $\alpha$ and $\beta$ are constant or their shift with $N^{\delta}$ is negligible, as it follows from the above definitions and derived relations ( $^{*}$. It is usual to regard these quantities the properties of the standard state of the ideal solution where $N^{\delta}=1$. Here we should rather treat these quantities as the properties of our homogeneous solution itself, if ideal or not, than have recourse to the standard state.

(*) $\bar{F}_{V, 1}^{\delta},\left(\frac{\partial \bar{F}_{V, 1}^{\delta}}{\partial P}\right)_{T}$ and hence $\bar{V}_{P}^{\delta}$ are constant according to Eq. (14.17.P) independent of $N^{\delta}$. Hence it follows from other relations the same is true with all quantities suffixed with 1 . 
In the case of a gas the independency of $\alpha, \beta$ and hence of $\bar{V}_{P}^{\delta}$ is strictly secured with the following particular values as derived from Eq. (14.15),

$$
x=\frac{1}{T}, \quad \beta=\frac{1}{P}, \quad \bar{V}_{P}^{\delta}=\frac{R T}{P} \quad(14.26 . \alpha),(14.26 . \beta),(14.26 . \mathrm{V})
$$

Eqs. (14.17), (14.20), (14.21), (14.24) and (14.25) assume now the particular forms :-

$$
\begin{aligned}
& \text { (14.17) } \quad \mu_{1}^{\delta}=\bar{F}_{V, 1}^{\delta}=\bar{F}_{P, 1}^{\delta}+R T=\bar{Z}_{P}^{\delta} \\
& \text { (14.20) } \quad \bar{S}_{V \cdot 1}^{\delta}=-\left(\frac{\partial \mu_{1}^{\delta}}{\partial T}\right)_{V}=\bar{S}_{P, 1}^{\delta}-R=-\left(\frac{\partial \mu_{1}^{\delta}}{\partial T}\right)_{P}
\end{aligned}
$$

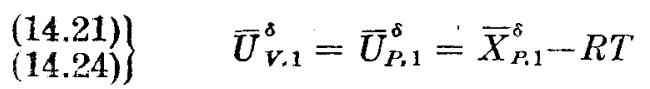

$$
\begin{aligned}
& \text { (14.25) } \quad\left(\frac{\partial \bar{X}_{P 1}^{\hat{A}}}{\partial P}\right)_{P}=0
\end{aligned}
$$

\section{CHAPTER III}

\section{APPLICATION-1 EQUILIBRIUM}

\section{$\$ 15$. Bose-Einstein and Fermi-Dirac Statistics.}

Assuming both Bose-Einstein and Fermi-Dirac gas are as a whole describable by an appropriate petit quantum canonical ensemble, the mean population or the distribution function $f_{s}$ of quantum state $\sigma_{s}$ of energy $\xi_{8}$ for individual molecules $\delta$ 's is given by

$$
f_{s}=\frac{0 \times \boldsymbol{\theta}_{\sigma_{s}(0)}+1 \times \boldsymbol{\theta}_{\sigma_{s}(\delta)}+2 \times \boldsymbol{\theta}_{\sigma_{s}(2 \delta)}+3 \times \boldsymbol{\theta}_{\sigma_{s}(8 \delta)}+\cdots}{\boldsymbol{\theta}_{\sigma_{s}(0)}+\boldsymbol{\theta}_{\sigma_{s}(\delta)}+\boldsymbol{\theta}_{\sigma_{s}(2 \delta)}+\boldsymbol{\theta}_{\sigma_{\tilde{s}}(8 \delta)}+\cdots}
$$

where $\boldsymbol{\theta}_{\sigma_{s}(\nu \delta)}$ denotes the probability that $\nu$ pieces of $\delta$ 's simultaneously occupy the elementary state $\sigma_{s}$, which vanishes for $\nu>1$ according to the Pauli principle in the case of Fermi-Dirac gas.

$\boldsymbol{\theta}_{\mathrm{o}_{\boldsymbol{s}}(\nu \delta)}$ in the case of Bose-Einstein gas may be written according to the definitions Eqs. (2.3) and (2.4) that, 


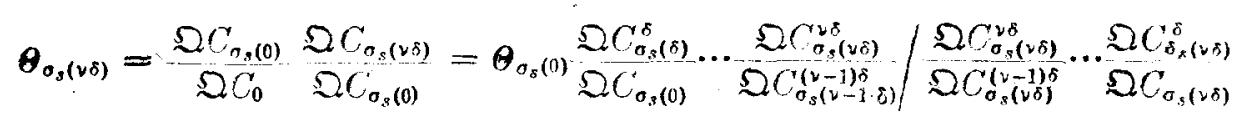

The denominator of the third member of Eq. (15.2) simply oquals $\left(p^{\delta}\right)^{\nu}$ according to the approximation of $\$ 4$, provided that $\nu$ is not very large.

The first factor of the numerator equals $q_{\sigma_{s}}^{\delta}$ according to Eq. (2.2), i.e.

$$
q_{\sigma}^{\hat{\delta}}=\frac{\mathfrak{Q} C_{o_{s}(\delta)}^{\delta}}{\mathfrak{Q} C_{\sigma_{s}(0)}}
$$

which may be evaluated as follows : sinco Bose-Einstein statistics attributes the same statistical weight to every possible distribution of $N$ molecules in the gas over all elementary states and to the total energy the sum $\sum_{t} \nu_{t} \varepsilon_{t}$ of individual energies $\varepsilon_{t}$ 's of all molecules respectively at quantum states $\sigma_{t}$ 's, $\mathfrak{Q} C_{\sigma_{s}(0)}$ is the summation of Boltzmann factors of $\sum_{t} \nu_{t} \varepsilon_{t}$ over all possible distribution of $N$ molecules with the constraint $\nu_{s}=0$ i.e. that of $N$ molecules over all $\sigma_{t}$ 's except $\sigma_{s} . \mathfrak{D} C_{\delta_{s}(\delta)}^{\delta}$ is similarly that of $N+1$ molecules with the constraint $\nu_{s}=1$ or that of $N$ molecules over all $\sigma_{t}$ 's except $\sigma_{s}$ which holds $N+1-$ th $\delta$. It follows that there is one to one correspondence between Boltzmann factors of the two series relevant respectively to $\mathfrak{Q} C_{\sigma(\delta)}^{\delta}$ and to $\mathfrak{Q} C_{\sigma(0)}$ so that every one of the former series differs from the corresponding one of the latter by a factor $e^{-\frac{\varepsilon_{0}}{c c T}}$. We have thus,

$$
\mathfrak{Q} C_{\boldsymbol{o}_{s}(\delta)}^{\delta}=\mathfrak{Q} C_{\sigma_{s}(0)} e^{-\frac{\varepsilon_{s}}{k T^{*}}}
$$

or according to Eq. (15.3),

$$
q_{\mathbf{s}_{s}}^{\hat{\delta}}=e^{-\frac{\varepsilon_{s}}{k T}}
$$

It is shown similarly the remaining factors of the numerator equals $q_{\text {s }}$. or $e^{-\frac{e_{s}}{k T}}$ so far as the premise of the Bose-Einstein statistics gces.

We have hence in place of Eq. (15.2), that,

$$
\boldsymbol{\theta}_{\sigma_{s}(2 \delta)}=\left(q_{o_{s}}^{8} / p^{8}\right)^{2} \boldsymbol{\theta}_{o_{s}(0)}
$$

or substituting $\boldsymbol{\theta}_{\boldsymbol{s}_{s}(\mathrm{v})}$ from Eq. (15.6) into Eq. (15.1), 
A Method of Statistical Mechanical Treatment of Equilibrium and Chemical Reactions.

$$
f_{s}=\frac{0 \times 1+1 \times\left(q_{\sigma_{s}}^{\delta} / p^{\delta}\right)+2 \times\left(q_{\sigma_{s}}^{\delta} / p^{\delta}\right)^{2}+\cdots}{1+\left(q_{\sigma_{s}}^{\delta} / p^{\delta}\right)+\left(q_{\boldsymbol{o}_{s}}^{\delta} / p^{\delta}\right)^{2}+\cdots}
$$

Owing to the above approximation $f_{s}$ is given by Eq. (15.7) only when the power series of the numerator and denominator converges or $\left(q_{\sigma_{s}}^{\delta} / p^{\delta}\right)^{\nu}$ for large $\nu$ is insignificant. In that case when $q_{\sigma_{s}}^{\delta} / p^{\delta}<1 \mathrm{Eq}$. (15.7) is written in the form,

$$
f_{\mathbf{s}}=\frac{q_{\sigma_{s}}^{\delta} / p^{\delta}}{1-q_{\sigma_{s}}^{\delta} / p^{\delta}}
$$

or expressing $q_{\sigma_{s}}^{\delta}$ by Eq. (15.5) and $p^{\delta}$ by the corresponding reversible work $\bar{\varepsilon}=-k T \log p^{\delta}$,

$$
f_{s}=\left(e^{\frac{s_{s}-\bar{s}}{k T}}-1\right)^{-1}
$$

Remembering, on the other hand, that $\theta_{\left.\sigma_{s}(v)\right)}=0$ for $\nu>1$ in the case of Fermi-Dirac gas, we arrive, by a similar reasoning but without resorting to the approximation as above, at the distribution function,

$$
f_{\mathrm{s}}=\left(e^{\frac{\varepsilon_{\varepsilon}-\bar{\varepsilon}}{k T}}+1\right)^{-1}
$$

§16. Homogeneous Equilibrium.

The equilibrium relation $\mathrm{Eq}$. (6.2) readily affords the equation,

$$
\stackrel{a}{I}\left(\frac{Q^{\delta_{a}^{A}}}{\dot{N}^{\partial_{a}^{A}}}\right)^{\nu_{a}^{A}}=\stackrel{a}{I}\left(\frac{Q^{\delta_{b}^{B}}}{N^{\delta_{b}^{B}}}\right)^{\nu_{b}^{B}}
$$

for the equilibrium between two sets of components,

$$
\delta^{A}=\sum_{a} \nu_{a}^{A} \delta_{a}^{A} \text { and } \delta^{B}=\sum_{b} \nu_{b}^{B} \delta_{b}^{B}
$$

in a homogeneous fluid by substituting $p^{\hat{\nu}}$ s from Eq. (5.5), or

$$
K_{N}=\frac{\stackrel{b}{I}\left(N^{\delta_{b}^{B}}\right)^{\nu B}}{\stackrel{a}{I}\left(N^{\delta_{a}^{A}}\right)^{\nu_{a}^{A}}}=\frac{\stackrel{b}{I}\left(Q^{\delta_{b}^{B}}\right)^{\nu_{b}^{B}}}{\stackrel{a}{I}\left(Q^{\delta_{a}^{A}}\right)^{\nu A}}
$$

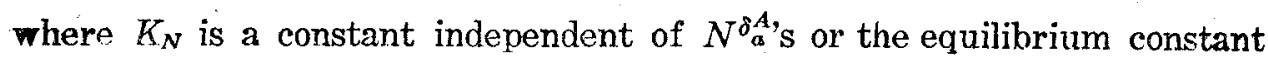
provided that $\delta_{a}^{A}$ s are all ideal components ${ }^{(*)}$.

(*) See p. 27 . 


\section{\$17. Heterogereous Equilibrium-1.}

We treat in this section the simplest "case of heterogeneous equilibrium i.e. the equilibrium between molecules of one kind adsorbed each on one of identical cites on a crystal surface and the molecules of the same kind in a homogeneous fluid. Our model of the crystal surface consists in general of small segments of lattice planes, each lattice point or a ceitain set of lattice points on any one of them providing a cite $\sigma^{A}$ for an adsorbed molecule where $Q^{\delta}$ or $N^{\delta}$ in Eq. (5.5) is concentrated, each segment being however big enough so that geometrically congruent $\sigma^{A}$ 's on that segment are practically physically identical.

Because of the equilibrium we have according to Eq. (6.2) that,

$$
p^{\delta_{t}}=p^{\delta_{h}}
$$

where $\delta_{a}$ or $\delta_{h}$ denotes adsorbed molecule or that in the homogeneous fluid respectively. Expressing $p^{\delta_{r}}$ by Eq. (5.1) and $p^{\delta_{h}}$ by Eq. (5.5), we have, dropping subscripts $a$ and $h$,

$$
\frac{\boldsymbol{\theta}_{o(\delta)}}{\Theta_{o(0)}}=q_{\circ}^{\delta} \frac{N^{\delta}}{Q^{\delta}}
$$

If any cite is either occupied by $\delta_{a}$ or else evacuated with certainty, we have,

$$
\theta=\theta_{\sigma(\varepsilon)}=1-\Theta_{o(0)}^{(*)}
$$

where $\theta$ is the degree of adsorption identified with $\theta_{o(\delta)}$.

Eliminating $\boldsymbol{\theta}_{\sigma(\delta)}$ and $\boldsymbol{\theta}_{\sigma(0)}$ from Eqs. (17.1) and (17.2), we have;

$$
\frac{\theta}{1-\theta}=\frac{q_{\sigma}^{\delta}}{Q^{\delta}} N^{\delta}
$$

Introducing a further approximation that each molecule adsorbed on a cite behaves like that in the homogeneous fluid in a cavity of the magnitude $|\sigma|$, within which the potential energy being constantly lower than that in the homogeneous fluid by $\epsilon_{\sigma}$, we have by similar reasoning as in the case of Eq. (5.4), that,

(*) This can not be the case when $\delta$ is allowed to occupy any eite which partly overlaps with $a$. 
A Method of Statistical Mechanical Treatment of Equilibrium and Chemical Reactions.

$$
q_{\sigma}^{\delta}=|\sigma| Q^{\delta} e^{\frac{\varepsilon_{0}}{k T}}
$$

Substituting the above expression in Eq. (17.5), the latter assumes a more familiar form,

$$
\frac{\theta}{1-\theta}=|\sigma| e^{\stackrel{2 o}{k} \bar{T}} N^{\delta}
$$

which gives the Langmuir's adsorption isotherm.

\$18. Heterogeneous Equilibrium-2.

We proceed in this section to a bit more complicated case when $\delta$ in the homogeneous fluid splits, when adsorbed, into two parts $\delta_{1}$ 's each occupying a cite as an adsorbed molecule in the foregoing example. The equilibrium relation Eq. (6.2) is now

$$
p^{\delta}=\left(p^{\delta}\right)^{2}
$$

which gives, when $p^{\bar{\delta}}$ and $p^{\delta_{1}}$ are substituted respectively from Eqs. (5.5) and (5.1), the relation,

$$
\frac{\Theta_{\sigma(0)}}{\theta_{\sigma(\delta)}} q_{o}^{\delta}=\sqrt{\frac{Q^{\delta}}{N^{\delta}}}
$$

Eliminating $\Theta_{\sigma(0)}$ and $\Theta_{\sigma(\delta)}$ from $\mathrm{E}_{q}$. (17.2) and (18.1) as in the foregoing section, we have,

$$
\frac{1-\theta}{\theta} q_{o}^{\delta}=\sqrt{\frac{Q^{\delta}}{N^{\delta}}}
$$

\section{\$19. Heterogeneous Equilibrium-3.}

In this section we treat another case, when any one of identical cites is either vacant or occupied by any one of molecules of the several components of a homogeneous fluid.

We have immediately that,

$$
\Theta_{\sigma(0)}+\sum_{i} \Theta_{\sigma\left(\delta_{i}\right)}=1
$$

whereas for individual $\delta_{i}$ similarly as in the case of $\overrightarrow{\mathrm{Eq}} \cdot(17.3)$. that, 


$$
\frac{\Theta_{\sigma\left(\delta_{i}\right)}}{\Theta_{\sigma(0)}}=q_{\sigma}^{\delta_{i}} \frac{N^{\delta_{i}}}{Q^{\delta_{i}}}
$$

and henceforth from Eqs. (19.1) and (19.2), that,

$$
\theta_{i}=\theta_{\sigma\left(\delta_{i}\right)}=\frac{q_{\sigma}^{\delta_{i}} N^{\delta_{i}} / Q^{\delta_{i}}}{1+\sum_{i} q_{\sigma}^{\delta_{i} i} N^{\delta_{i}} / Q^{\delta_{i}}}
$$

where $\theta_{i}$ is the degree of adsorption of $i$-th component identified with $\theta_{\sigma\left(\delta_{i}\right)}$.

Substituting the approximate expression for $q_{a}^{\delta_{i}}$ from Eq. (17.4) we have Eq. (19.3) in the form,

$$
\theta_{i}=\frac{|\sigma| N^{\delta_{i}} e^{-\frac{\varepsilon_{i}}{k T}}}{1+\sum_{i} ! \sigma \mid N^{\delta_{i}} e^{-\frac{\varepsilon_{i}}{k} T^{-}}} .
$$

where $\varepsilon_{o_{i}}$ is $\varepsilon_{o}$ for $\delta_{i}$. An expression identical with Eq. (19.4) is arrived at by Hückei ${ }^{(1)}$ by a special reasoning.

\section{$\$ 20$. Heterogeneous Equilibrium-4.}

In this section we develop a method of deriving the adsorption isotherm allowing for the mutual interaction among adsorbed molecules.

Our method will first be exemplified with the case when the molecules $\delta$ 's of only one kind from a homogeneous fluid are adsorbed in equilibrium each on one of the identical cites $\sigma$ 's allayed on a plane square 'pattern, the interaction being significant only between directly neighboring adsorbed molecules.

We denote our assembly, consisting of the adsorbent attached with a definite number of $\delta$ 's and the homogeneous fluid, by $C$ with appropriate subscripts: $C_{\sigma_{0}(0)}$ or $C_{\sigma_{0}(\delta)}$ thus denotes that with its particular cite $\sigma_{0}$ evacuated or occupied respectively, $C_{\sigma_{s}(0)}$ or $C_{\sigma_{s}(\delta)}$ that with $\sigma_{s}$, one of four direct neighbors of $\sigma_{0}$ numbered by $s=1,2,3$ or 4 , evacuated or occupied respectively, $C_{\Sigma(0)}$ that with the set of all five cites $\sigma_{0}$ and $\sigma_{s}$ 's, denoted by $\Sigma$, are evacuated, and finally $C_{0}$ that without any such constraint.

$\mathfrak{S C _ { 0 }}$ is now expressed in terms of $C_{\mathrm{\Sigma}(0)}, p^{\delta}, q_{\boldsymbol{\sigma}(0)}^{\hat{\delta}}, \xi$ and $\eta$, the latter

(1) Hückel ; “Adsorption und Kapillarkondensation", Leipzig 1928, "p. 217. 
A Msthod of Statistical Mechanical Treatment of Equilibrium and Chemical Reactions.

two as described below taking care of the mutual interaction, as that,

$$
\mathfrak{D C} C_{0}=\mathfrak{\Omega} C_{\Sigma(0)} \sum_{t_{1}, t_{1}, t_{2}, t_{3}, t_{4}}\left(\frac{q_{6,0}^{\delta}}{p^{\delta}}\right)^{t_{0}+t_{1} \div t_{2}+t_{3}+t_{4}} \xi^{t_{0}\left(t_{1}+t_{2}+t_{3}+t_{3}\right)} \eta^{t_{1}+t_{9}+t_{3}+t_{4}}
$$

where $t_{0}$ etc. denote respectively 1 or 0 , according as $\sigma_{0}$ etc. is ocupied or not, $\sum_{t_{0}, t_{1}, t_{2}, t_{3}, t_{3}}$ the summation over all possible sets of values of $t_{0}$ etc.

We see with regard to $\mathrm{Eq} .(20.1)$ that $\frac{q_{\sigma, 0}^{\delta}}{p^{\delta}}$ would give the factor of multiplication of $\mathrm{Zs}$ when one of $\delta$ is picked up from outsida and planted on any vacant cite of $\sum$, and hence $\sum_{t_{0} \cdots t_{4}}\left(\frac{q_{0,0}^{\delta}}{p^{\delta}}\right)^{t_{0}+t_{3}+t_{2}+t_{3}+t_{4}}$ the

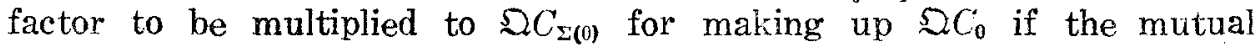
interaction were absent. The factor $\xi$ or $\eta$ gives now the extra factor of multiplication either due to the interaction of each adjacent pair of adsorbed molecules inside $\Sigma$, or due to the interaction between one of them and others outside $\sum$ respectively. They may differ from unity except for $\sigma_{0}$ and is the same for $\sigma_{1}, \sigma_{2}, \sigma_{3}$ and $\sigma_{4}$ because of the symmetry of $\sum$. Carrying out the summation we have,

$$
\mathfrak{D C} \boldsymbol{C}_{0}=\mathfrak{D} C_{\Sigma(0)}\left(1+q_{\sigma, 0}^{\delta} \eta / p^{\delta}\right)^{4}+\mathfrak{D C} C_{\Sigma(0)} \frac{q_{a, 0}^{\delta}}{p^{\delta}}\left(1+q_{\sigma, 0}^{\delta} \eta \xi / p^{\delta}\right)^{4}
$$

$\mathfrak{D} C_{\sigma_{g}(0)}$ or $\mathfrak{D C} C_{o_{0}(\delta)}$ is given respectively by the first or the second term on the right hand side of $\mathrm{Eq} .(20.2)$, which is the part of the summation of Eq. (20.1) respectively corresponding to $t_{0}=0$ or $t_{0}=1$, i.e.,

$$
\begin{aligned}
& \mathfrak{D} C_{\sigma_{\theta}(0)}=\mathfrak{D} C_{\Sigma(0)}\left(1+q_{\sigma, 0}^{\delta} \eta / p^{\delta}\right)^{4} \\
& \mathfrak{D} C_{\sigma_{\theta}(\delta)}=\mathfrak{Q} C_{\Sigma(0)} \frac{q_{\sigma, 0}^{\delta}}{p^{\delta}}\left(1+q_{\sigma, 0}^{\delta} \eta \xi / p^{\delta}\right)^{4}
\end{aligned}
$$

$\mathfrak{S C} C_{\sigma_{s}(\theta)}$ is given as the part of the sum corresponding to $t_{s}=0$ as that,

$$
\mathfrak{D} C_{\sigma_{s}(0)}=\mathfrak{Q} C_{\Sigma(0)}^{\delta}\left\{\left(1+\frac{q_{\sigma, 0}^{\delta} \eta}{p^{\delta}} \eta\right)^{8}+\frac{q_{\sigma, 0}^{\delta}}{p^{\delta}}\left(1+q_{\sigma, 0}^{\delta} \eta \xi / p^{\delta}\right)^{3}\right\}
$$

The degree of adsorption $\theta$ or $\boldsymbol{\theta}_{\sigma_{0}(\delta)}$ is now given according to Eos. (2.3), (20.2) and (20.4) in the form, 


$$
\theta=\boldsymbol{\theta}_{o_{0}(\delta)}=\frac{q_{a, 0}^{\delta}\left(1+\frac{q_{\sigma, 0}^{\delta} \eta \xi}{p^{\delta}}\right)^{4}}{\frac{p^{\delta}}{q_{\sigma .0}^{\delta}}\left(1+\frac{q_{\sigma, 0}^{\delta} \eta \xi}{p^{\delta}}\right)^{\alpha}+\left(1+\frac{q_{\sigma, 0}^{\delta} \eta}{p^{\delta}}\right)^{4}}
$$

We now deduce in accordance with Bethe and Peierls ${ }^{(*)}$ that $\mathfrak{\Omega C _ { \sigma _ { 0 } ( 0 ) }}$ must equal $\Omega C_{\sigma_{3}(0)}$, since $\sigma_{0}$ and $\sigma_{s}$ are physically identical, i.e. according to Eqs. (20.3) and (20.5) that,

$$
\left(1+q_{\sigma, 0}^{\delta} \eta / p^{\delta}\right)^{4}=\left(1+q_{\sigma, 0}^{\delta} \eta / p^{\delta}\right)^{3}+q_{\sigma, 0}^{\delta} / p^{\delta}\left(1+q_{\sigma, 0}^{\delta} \eta \xi / p^{\delta}\right)^{3}
$$

Eliminating $y$ from Eqs. (20.5) and (20.7) we have $\theta$ as a function of $p^{\delta}, q_{\boldsymbol{\sigma}, 0}^{\delta}$ and $\xi$.

The $p^{\delta}$ is given by the equilibrium relation as in $\$ 17$ and $\$ 18$ as the function of 'the concentration or pressure in' the homogeneous fluid.

The $q_{\sigma, 0}^{\delta}$ is according to the definition is given as the ratio of $\mathfrak{Q} M_{0}^{\delta}$ to $\mathfrak{D} M$, where $M_{o}^{\delta}$ is the adsorbent in the fluid with only one $\delta$ adsorbed at $\sigma$ and $M$ the latter without adsorbed $\delta$.

With the approximation that the $\delta$ and the rest of the assembly are each at thermal motion reciprocally in the mean potential field of the other and that the height of energy levels of $M$ proper above the energy at rest remain unchanged by adsorbing $\delta, \mathfrak{Q} M_{a}^{\delta}$ may be expressed by factors as that,

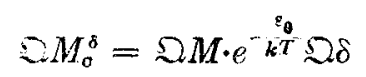

and hence we have for $\varphi_{\sigma, 0}^{\delta}$,

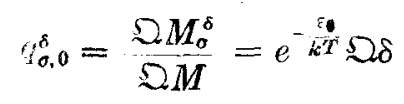

where $2 \delta$ is the $Z s$ of $\delta$ moving in the mean potential field of $M$ or the sum of Boltzmann factors of Eigenwerts referred to the minimum $\epsilon_{3}$ of the mean potential.

The factor $\xi$ is estimated again with the approximation that each of the two admorbed molecules interacting moves reciprocally in the mean potential field of the other, the height of individual energy levels above

(*) Bethe; Proc. Roy. Soc. London A 150 (1935), 552. Peierls; Proc. Cambridge Phil. Soc. 32 (1936), 471. 
A Method of Statistical Mechanical Treatment of Equilibrium and Chemical Reactions.

the minimum potential energy remaining unaffected by the interaction. The interaction contributes thus simply a constant term $u$ to the Eigenwerts of the whole assembly or a constant factor $\xi=e^{-\frac{\boldsymbol{q}}{\boldsymbol{k} T}}$ to the Zs. The $\xi$ may thus be obtained by evaluating $u$ propely.

Okamoto, Horiuti and Hirota ${ }^{(*)}$ estimated $q_{c, 0}^{\delta}$ and $\xi$ after this manner in developing their theory of the hydrogen electrode process.

The present method may readily be extended to the case when the pattern of cites is of less symmetry and the allowence is made for the interaction between remoter molecules than directly neighboring. Attributing different extrafactor of multiplication $\eta$ to every symmetry class of $\sigma_{s}$ s appropriate to respective symmetry operation with respect to $\Sigma$ and different $\xi$ to every pair of cites of different relative position in $\Sigma$, $\mathfrak{D C} C_{\sigma_{0}}, \mathfrak{Q} C_{\sigma_{\delta}(\theta)}, \mathfrak{Q} C_{\sigma_{s}(\delta)}$ and $\mathfrak{Q} C_{\sigma_{0}(\delta)}$ are given by $\mathfrak{D C} C_{\Sigma(0)}$ multiplied by a function of $p^{\delta}, q_{\sigma \theta}^{\delta}, \eta$ 's and ' $\xi$ 's.

The degree of adsorption $\theta$ is given as in the previous cases by

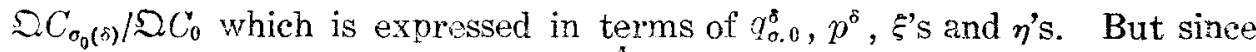
we have so many different expressions for $\mathfrak{Q} C_{\sigma_{s}(0)}$ as the number $g$ of symmetry classes of $\sigma_{s}$ 's as well as $\mathfrak{\Omega} \widehat{C}_{\sigma_{0}(0)}$ which are to be set equal to each other, we have $g$ independent relations among $\mu$ 's, $p^{\delta}, q_{\sigma, 0}^{\delta}$ and $\xi^{\prime}$ 's. Solving $\eta$ 's therefrom and substituting the latter into the expression for $\theta$, we have $\theta$ in terms of $p^{\hat{\delta}}, q_{0,0}^{\hat{\sigma}}$ and $\xi^{\prime}$ 's. Since $p^{\hat{\delta}}$ is in turn determined by the equilibrium relation, we obtain estimating $q_{\sigma, 0}^{s}$ and $\xi$ 's properiy as in the previous case, $\theta$ as a function of the concentration of molecules in the homogeneous flaid i.e. the adsorption isotherm.

An actual calculation by the method outlined above will be presented in later papers.

\section{CHAPTER IV}

\section{APPLICATION-II. CHEMICAL REACTION IN GENERAL.}

821. The Scope and Procedure of Application.

At the present stage of the chemical kinetics a reaction is taken as the over-all result of a sequence of elementary reactions, the sequence, which adequately describes the experimental result in comformity with 223.

(*) Okakoto, Horiuti and Hirota; Sc. Pap. Inst. Phys. Chem. Res. Tokio, 29 (1936) 
the stoichiometrical relation or the chemical equation, being called its mechanism. According to the postulate is our procedure now the statistical mechanical description of the individual elementary reactions and hence of the over-all rate synthesizing the latters.

Another postulate underlying to the current procedure in the chemical kinetics is that the rate of elementary reactions is determined at a given temperature and external parameters solely by the momentary chemical composition of the assembly involved not depending on the history or explicitly on time. We might advance amplifying the latter a postulate in its place in detailed statistical mechanical terms thus affording an exact basis for the application of our procedure.

Consider an assembly which would be derived from the observed one if left standing under the preseribed external condition to attain an equilibrium. The elementary reaction will be there going on in either direction more or less fluctuating the chemical composition.

The canonical ensemble of the assembly at equilibrium will of course consist of overwhelming majority of examples of the chemical composition close to the average at equilibrium, but a few, if exceptional, will have the chemical composition of the observed assembly, where so-called irreversible reaction is in general in progress. We postulate now that the chance with which a particular elementary reaction occur to the assembly as well as its momentary properties is given as the average over that part of the ensemble which happened to have the momentary chemical composition of the assembly.

We call the assembly in thermal equilibrium if thus describable and the corresponding part of Zs that of the assembly. In terms of the Zs we define $\lambda$ 's of the assembly just as in the case of those in complete equilibrium. Taking the fixed chemical composition as the rnicroscopic constraint the whole theory of $\lambda$ developed in Chapter II is applicable for the assembly at thermal equilibrium.

After we have defined in the next section the reaction of the particular type we are going to deal with, we advance in $\$ 23$ the expression for the rate of the elementary reaction at thermal equilibrium derived on the basis of the above postulate.

The over-all chemical reaction to be composed of elementary reactions is generally expressed by,

$$
\begin{aligned}
\delta^{L} & =\delta^{R} \\
& -42-
\end{aligned}
$$


A Method of Statistical Mechanical Treatment of Equilibrium and Chemical Reactions.

where $\delta^{L}$ or $\delta^{R}$ denotes reactant or resultant consisting generally of each $\nu_{i}^{L}$ pieces of $\delta_{l}^{L}$ or of each $\nu_{r}^{R}$ pieces of $\delta_{r}^{R}$, so that,

$$
\delta^{L}=\sum_{l} \nu_{l}^{L} \delta_{l}^{L}, \quad \delta^{R}=\sum_{r} \nu_{r}^{R} \delta_{r}^{R} \quad(21.2 . \mathrm{L}),(21.2 . \mathrm{R})
$$

We call, on the other hand, the set of elementary particles involved in the elementary reaction generally the reaction complex and that at the state before or after the elementary reaction in particular the initial or final complex in distinction respectively from the reactant or resultant of the over-all reaction. The initial and the final compiex are in general taken as consisting respectively of each $\nu_{i}^{I}$ pieces of molecules $\delta_{i}^{I}$ and each $\nu_{f}^{F}$ ones of $\delta_{f}^{F}$ so that,

$$
\delta^{I}=\sum_{i} \nu_{i}^{I} \delta_{i}^{I}, \quad \delta^{F}=\sum_{f} \nu_{f}^{F} \delta_{f}^{F} \quad \text { (21.3.1), (21.3.F) }
$$

The simplest mechanism is that of the reaction consisting of only one elementary reaction. The reaction of this mechanism will be called the simple reaction. In general, however, the sequence needs not even be a consecutive one but branched, multiple one so that geveral different courses are available for a reactant to pass into the resultant. The type of sequences or the mechanism of the reaction going to be dealt with is that, inclusive of the simple reaction, of the steady reaction with a rate-determining step, proceeding at thermal equilibrium.

In $\$ 24$ we express the rate in terms of $\lambda$ 's. The expression is now ready for various particular applications in later sections.

\section{\$22. The Steady Reaction with Rate-Determining Step at Thermal Equilibrium.}

It is meant by steady reaction such one as that the rates of creation and consumption of every intermediate product are practically balanced and by the rate-determining step an elementary reaction which is necessary for the reaction to proceed at all and has a negligibly small rate in either direction compared with that of any other constituent elementary reaction of the reaction.

The following may be inferred from thus detailed definition.

(i) The initial complex of the rate-determining step of the reaction is most probably transferred into the reactant rather than into the final complex, and the final complex into the resultant rather than into the 
reactant or in other word that an initial complex of the rate-determining step originates practically all in the reactant and the final complex practically certainly pass into the resultant, and hence that one act of the rate-determining step is necessary and sufficient for the reaction complex of the rate-determining step to complete the reaction i.e. to pass from the state of the reactant to that of the resultant.

(ii) The excess of the rate of any elementary reaction in one direction over that in the reversed direction must be equal to or smaller ${ }^{* 1}$ than that of the rate-determining step for the reaction to proceed steadily or without accumulation of the intermediate product. But since the rate in either direction of the rate-determining step itself is negligible compared with either of any other elementary reaction, the same must be true with the excess. It follows that for any other elementary reaction than the rate-determining one the excess is negligibly small compared with its rate in either direction, or in other word both the rates are practically balanced.

\section{\$23. The Rate of the Elementary Reaction.}

The rate of elementary reaction which proceeds adiabaticaliy inside an assembly at thermal equilibrium is derived as follows:(1)(2)

$$
i=\kappa \frac{k T}{h} \frac{\mathfrak{S C} C_{0}^{\delta^{*}}}{\mathscr{S C} C_{0}^{\delta^{I}}}
$$

The $\mathscr{Q} C_{0}^{\delta^{*}}$ or $\Omega C_{0}^{\delta^{I}}$ is the classical approximation of the $\mathrm{Zs}$ of the assembly $C_{0}^{\delta^{*}}$ or $C_{0}^{\delta^{T}}$ i.e. of the assembly $C_{0}$ of definite chemical composition augmented by the critical complex $\delta^{*}$ or by the initial complex $\delta^{I}$.

$C_{0}^{\delta^{*}}$ is defined as $\overline{C_{0}^{\delta}}$ at a particular configulation corresponding to the minimum of $\mathfrak{S} C_{0}^{\bar{\delta}}$, where $C_{0}^{\bar{\delta}}$ is an assembly consisting of $C_{0}$ of definite chemical composition and the reaction complex $\bar{\delta}$ within the latter with its representative point in the conffulation space resting on a hypersurface separating the region corresponding to the state of $\delta^{I}$ of $\bar{\delta}$ from that of $\delta^{F}$. The hypersurface thus determined is called the

(*) The excess may be smaller than that of the rate-determining step when the course is branched.

(1) Horiuti ; Bull. Chem. Soc. Japan, Katayama Commem. Vol. 13 (1938), 210.

(2) Hirota and Horiuti; Sc. Pap. Inst. Phys. Chem. Res. 34 (1938), 1174. 
critical surface and $\vec{\delta}$ at the corresponding particular state the critical complex $\delta^{*}$.

The transmission coefficient $\kappa$ in Eq. (23.1) is defined as follows : we consider the representative points of the part of canonical ensemble, referred to in $\$ 22$, appropriate to the chemical composition of the assembly $C^{\bar{\delta}}$. Because of the microscopic reversibility, however, the flow of the representative points through the critical surface is balanced by the reversed one. The transmission coefficient $\kappa$ is now the ratio of the number of representative points which complete the elementary reaction over that which transit the critical surface in either direction. It follows from the definition of $\mathfrak{Q} C_{0}^{\delta^{*}}$ and $\kappa$ the latters are the same for the reverse elementary reaction. The rate $t$ of the reverse elementary reaction is hence given by,

$$
\hat{v}=\kappa \frac{k T}{h} \frac{\mathfrak{Q} C_{0}^{\delta^{*}}}{\mathfrak{Q} C^{\delta^{F}}}
$$

The derivation of the above expression has nothing to do with some equilibrium relation between the initial and the critical complex and is generally applicable to the assembly at thermal equilibrium not being restricted to the case of the reaction complex behaving dynamically independent. Nor it presupposes the existance of the saddle point of the potential energy and its being determinant of the rate. As shown later the expression thus derived includes that of Eyring(1) or of Evans and Polanyi ${ }^{(2)}$ as its special case.

\$24. Classification of Reaction and Different Expressions for the Rate.

Eqs. (23.1) and (23.2) for the rate of the elementary reaction may readily expressed according to Eq. (2.1) in terms of $\lambda$ 's, i.e.

$$
\vec{v}=\kappa \frac{k T}{h} \frac{p^{\delta^{*}}}{p^{\delta^{T}}}, \quad \overleftarrow{v}=\kappa \frac{k T}{h} \frac{p^{\delta^{*}}}{p^{\delta^{\delta^{*}}}}
$$

where

$$
p^{\delta^{I}}=\stackrel{i}{I}\left(p^{\delta_{i}^{I}}\right)^{\nu_{i}^{I}}, \quad p^{\delta^{H}}=\stackrel{f}{I}\left(p^{\delta_{f}^{F}}\right)^{\nu_{f}^{F}}
$$

(1) Eyring; J. Chem. Phys. 3 (1935), 107.

(2) Evans and Polanyi: Trans. Faraday Soc. 31 (1935), 875. 
according to Eqs. (4.1), (21.3.I) and (21.3.F). The function $p^{6^{*}}$ in Eqs. (24.1.) and (24.1.v) may either be expressed according to Eq. (5.1) in the form,

$$
p^{\delta^{*}}=\frac{\boldsymbol{\theta}_{o^{*}(0)}}{\boldsymbol{\theta}_{o^{*}(\delta)}^{\delta_{\sigma^{*}}^{*}}}
$$

or according to Eqs. (5.5) and (5.3) in the form,

$$
p^{\delta^{*}}=\frac{Q^{\delta^{*}}}{N^{\delta^{*}}}, \text { where } Q^{\delta^{*}}=\operatorname{Lim}_{|\sigma *| \rightarrow 0} \frac{q_{\sigma^{*}}^{\delta^{*}}}{\left|\sigma^{*}\right|}
$$

and $\sigma^{*}$ denotes a small cavity of molecular dimension housing the centre of gravity of $\delta^{*}$ within. Substituting $p^{6^{*}}$ from Eq. (24.3) or from (24.4) into (24.1), we have,

$$
\vec{v}=\kappa \frac{k T}{h} \frac{Q^{\hat{o}^{*}}}{N^{\delta^{*}} p^{\delta^{I^{-}}}}, \quad \overleftarrow{v}=\kappa \frac{k T}{h} \frac{Q^{\delta^{*}}}{N^{\delta^{*}} p^{\delta^{\hat{k}^{\prime}}}}
$$

or

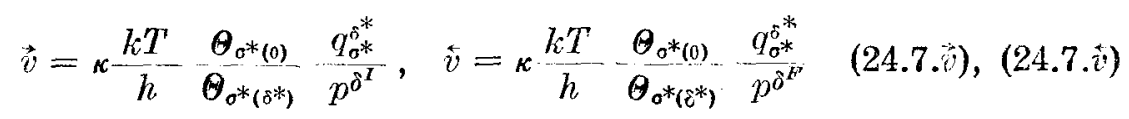

We call along with $\$ 14$ an elementary reaction homogeneous, if $Q^{\delta^{*}}$ and hence $N^{\delta^{*}}$ remain constant all over the space concerned, or heterogeneous, if $Q^{\delta^{*}}$ and hence $N^{\delta^{*}}$ are concentrated at cite $\sigma^{*}$ of the similar description as that of $\sigma^{A}$ in $\S 17$. A reaction will be called homogeneous or heterogeneous accoroing as its rate-determining step is either.

Eq. (24.6) may conveniently be transformed as follows for the homegeneous elementary reaction. $N^{\delta^{*}}$ being the concentration of only one $\delta^{*}$ exsisting in the assembly ${ }^{(*)}$, it may be identified with the reciprocal of volume $\mathrm{V}$ of the homogeneous fluid where $\delta^{*}$ is in. We obtain thus from Eq. (24.6), that,

$$
\vec{v}_{1}=\frac{\vec{v}}{V}=\kappa \frac{k T}{\grave{h}} \frac{Q^{\delta^{*}}}{p^{\hat{o}^{I}}}, \quad \dot{v}_{1}=\frac{\grave{v}}{V}=\kappa \frac{k T}{h} \frac{Q^{\delta^{*}}}{p^{\delta^{F}}}
$$

where $\vec{v}_{1}$ and $\hat{v}_{1}$ are respective rates per unit volume.

(*) Cf. $\$ 23$. 
Eq. (24.7) is on the other hand transformed for the heterogeneous elementary reaction occurring in identical cites $\sigma^{*}$ 's of total number $Z$. $\mathfrak{Q} C_{0}^{\delta^{*}}, p^{\delta^{*}}$ and $\Theta_{\sigma^{*}\left(\delta^{*}\right)}$ involved in the expression are, now in accordance with $\$ 23$, those of the assembly containing one $\delta^{*}$ in either one of $\sigma^{*}$. It follows that $G \Theta_{o^{*}\left(\delta^{*}\right)}=1$ or $\theta_{o *(\delta *)}=1 / G$ i.e. that Eqs. (24.7) assumes the form,

$$
\begin{aligned}
& \vec{v}_{1}=\frac{\vec{t}}{A}=\kappa \frac{k T}{h} G_{1} \frac{q_{\sigma^{*}}^{\sigma^{*}}}{p^{\delta^{J}}} \Theta_{\sigma^{*}(0)}, \\
& \hat{v}_{1}=\frac{\hat{v}}{A}=\kappa \frac{k T}{h} G_{1} \frac{q_{\sigma^{*}}^{\delta^{*}}}{p^{\delta^{F}}} \Theta_{\sigma *(0)}
\end{aligned}
$$

where $A$ is the area of the surface and $G_{1}$ is the number of $\sigma^{*}$ 's per unit area. The total rate may be obtained by simply summing up $\hat{v}_{1}$ or $\dot{v}_{1}$ of Eq. $(24.9 . \vec{v})$ or $(24.9 . \vec{v})$ with respect to all kinds of cites.

\section{CHAPTER V}

\section{APPLICATION-III. THE CHARACTERISTIC NUMBER OF THE REACTION}

\section{$\$ 25$. The Order of Reaction.}

The order $m$ of a reaction has hitherto been taken the characteristic number of the reaction and the most important information about the mechanism.

Let the homogeneous fluid of our assembly contain members $\delta_{l}^{L}$ 's of the reactant respectively at concentrations $N^{\delta} l$ 's but none of the resultant, the former passing over into the reactant at a rate $\vec{r}_{0}$ per unit volume of the fluid. The number $m$ is expressed as that,

$$
m=\sum_{l} \frac{\partial \log \vec{r}_{0}}{\partial \log N^{\partial_{l}^{L}}}
$$

The individual term $\frac{\partial \log \vec{r}_{0}}{\partial \log N^{\delta_{l}^{L}}}$ on the right-hand side of Eq. (25.1) gives what is called the order of reaction $m_{l}^{L}$ with respect to $\delta_{l}^{L}$ i.e. 


$$
m_{l}^{L}=\frac{\partial \log \vec{r}_{0}}{\partial \log N^{\delta_{l}^{L}}}
$$

We consider on the basis of rate expressions advanced in $\S 24$, how the order of reaction indicates the mechanism, first with the homogeneous simple reaction ${ }^{*)}$, assuming throughout that $\kappa$ remains constant with progress of the reaction.

We have, in this case according to the definition,

and

$$
\begin{aligned}
& \vec{r}_{0} \equiv \hat{\imath}_{1} \\
& \delta^{L} \equiv \delta^{I}, \quad \delta^{R} \equiv \delta^{F}
\end{aligned}
$$

$$
p^{\delta^{L}} \equiv p^{0^{I}}, \quad p^{\delta^{R}} \equiv p^{\delta^{F}}
$$

where $t_{1}$ is the rate of the elementary reaction reckoned per unit volume of the homogeneous fluid. Expressing $p^{\delta^{I}}$ and $p^{\delta^{R}}$ according to Eqs. (4.1) and (21.2) in the forms,

$$
p^{\delta^{L}}=\stackrel{l}{I}\left(p^{\delta_{i}^{L}}\right)^{\nu L}, \quad p^{\delta^{R}}=\stackrel{r}{I}\left(p^{\delta_{r}^{R}}\right)^{\nu_{r}^{R}}
$$

we have by Eqs. (25.3), (25.4.L), (25.5.L), (5.5) and (24.8.i), that.

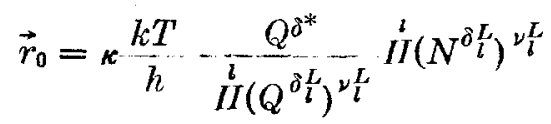

i.e. that $\vec{r}_{0}$ is proportional to $\stackrel{l}{I}\left(N^{\delta_{l}^{L}}\right)^{\nu_{l}^{L}}$ provided that components of variable concentration behave ideal so that $Q^{8 \prime}$ s remain constant in the course of the reaction. The order is now given according to Eqs. (25.1), (25.2) and (25.6) as that,

$$
m=\sum_{i} \nu_{l}^{L}
$$

and

$$
m_{l}^{L}=\nu_{l}^{L}
$$

The order of reaction $m_{l}^{L}$ with respect to $\delta_{l}^{L}$ hence equals the number of the molecule $\delta_{l}^{L}$ reacting and $m$ the total number of such molecules.

(*) see p. 43 . 
Conversely, if observed value of $m_{l}^{L}$ respectively equals the stoichiometrical coefficient $\nu_{i}^{L}$ of the chemical equation, the mechanism may well be that of the simple reaction but not necessarily.

In the case when the simple reaction is heterogeneous the same is true with $m$ and $m_{l}^{L}$, since $\vec{r}_{0}$ or $\vec{v}_{1}$ is, as in the previous case, proportional to ${ }^{l}\left(\frac{N^{\delta_{l}^{L}}}{Q^{\delta_{l}^{L}}}\right)^{\nu_{L}^{L}}$ in the homogeneous fluid according to Eqs. (25.3), (25.4.L), (25.5.L), (5.5) and (24.9. $)$ ), provided that $Q^{\delta L}$ 's and $q_{\sigma *}^{\delta *} \theta_{\sigma *(0)}$ in $\mathrm{Eq} .(24.9 . \vec{v})$ remains constant.

Passing now to the homogeneous reaction consisting of several elementary reactions, where every component of variable concentration behaves ideal, we first observe that $\vec{r}_{0}$ is proportional to $\vec{v}_{1}$ of the ratedetermining step, if not identical with, as in the case of simple reaction, that $\vec{v}_{1}$ in turn depends solely on $p^{\delta^{I}}$ of the step because of the ideality according to Eq. $(24.8 . \vec{v})$, that $p^{\delta^{I}}$ in turn is combined solely with $p^{\delta !}{ }^{I} \mathrm{~s}^{(*)}$ because of the equilibrium relation $\mathrm{Eq}$. (6.1) prevailing, according to $\$ 22$, (ii), with all other elementary reactions than the rate-determining step, and that $p^{\delta_{l}^{L}}$ in turn depends only upon $N^{\delta_{l}^{L}}$ according to Eq. (5.5). The order $m_{l}^{L}$ may hence be expressed according to Eq. (25.2) in the form,

$$
m_{l}^{L}=\frac{\partial \log \vec{r}_{0}}{\partial \log N^{\delta L}}=\frac{\partial \log \vec{r}_{0}}{\partial \log p^{\delta^{I}}} \cdot \frac{\partial \log p^{\delta^{I}}}{\partial \log p^{\delta_{l}^{L}}} \cdot \frac{\partial \log p^{\delta_{l}^{L}}}{\partial \log N^{\delta_{l}^{L}}}
$$

Both the first and third differential coefficient of the third member of the equation being -1 according to Eqs. $(24.8 . \vec{v})$ and $(5.5)$, we have,

$$
m_{l}^{L^{\prime}}=\frac{\partial \log p^{\delta^{I}}}{\partial \log p^{\delta_{l}^{L}}}
$$

which depends on the mechanism of the reaction.

In the case of the homogeneous para-ortho-conversion of hydrogen, if the rate-determining step is the elementary reaction,

(*) The form of the function $p^{\delta^{I}}=p^{\delta^{I}}\left(p^{\delta^{L}}, p^{8_{2}^{L}}, \ldots, p^{\delta^{L}}\right)$ depends on the mechanism. 


$$
H+p-H_{2} \rightarrow 0-H_{2}+H
$$

we have between $\delta^{I} \equiv p-H_{2}$ and $\delta^{I} \equiv H+p-H_{2}$ the equilibrium relation,

and hence

$$
\left(p^{\mathfrak{p}-H_{\mathbf{g}}}\right)^{\frac{3}{2}}=p^{H} \cdot p^{p-H_{\mathfrak{a}}}
$$

$$
m=m_{l}^{L}=\frac{3}{2}
$$

The number $m$ or $m_{l}^{L}$, if determined experimentarily, gives thus a criterion for the mechanism.

The same is true with the heterogeneous reaction provided that component of variable concentration behaves ideal and $\sigma_{\sigma^{*}}^{* * \theta_{\sigma^{*}(0)}}$ in Eq. $(24.9 . \vec{v})$ remains sufficiently constant.

The above procedure of providing a criterion for the mechanism may be useful in the case of the homogeneous reaction, when we can often foresee the ideality on the ground of the small concentration of variable composition, but less in the case of the heterogeneous reaction when we can hardly be sure whether $q_{o}^{\delta^{*}} \theta_{\sigma^{*}(0)}$ is sufficiently constant before we learn anything about the mechanism(*).

\section{\$26. The Stoichiometrical Number of the Reaction.}

We first define the rate of the reaction in the forward direction as the number $\overrightarrow{\mathfrak{v}}$ of reactants passing over into the resultant per unit time. $\overrightarrow{\mathfrak{v}}$ may be experimentally detêrmined by distinguishing reactants originally present from those produced by the backward reaction, labelling the reactants or resultants, say, by means of isotopes. Since one act of the rate-determining step in the forward direction is necessary and sufficient for its reacting complex to pass from the state of the reactant into that of the resultant(**), we have

$$
\overrightarrow{\mu \mathfrak{b}}=\vec{v}
$$

where $\mu$ is the number of forward acts of rate-determining step required to transfer one reactant completely to a resultant.

(*) Cf. $\$ 34$.

(**) Cf. $\$ 22$, (i). 
A Method of Statistical Mechanical Treatment of Equilibrium and Chemical Reactions.

The backward rate $\overleftarrow{\mathfrak{v}}$ defined similarly bears a relation to $\hat{v}$ that,

$$
\mu \grave{b}=\grave{v}
$$

Numerical values of $\overrightarrow{\mathfrak{v}}$ or $\hat{\mathfrak{v}}$ hence depends, although the product $\mu \overrightarrow{\mathfrak{b}}$ or $\mu \overleftarrow{\mathrm{y}}$ is fixed; on the absolute value of the coefficient in the relevant chemical equation, where only the ratio among them needs to be fixed. We refer here for the sake of concreteness $\mu, \overrightarrow{\mathfrak{v}}$ and $\overleftarrow{\mathfrak{b}}$ to the smallest possible intergral coefficients. We will call the characteristic number of the reaction $\mu$ thus fixed the stoichiometrical number and proceed to express it in the form accessible to the experimental determination.

The observable resultant rate $\vec{r}$ of decrease of reactant is given by,

$$
\vec{r}=\overrightarrow{\mathfrak{b}}-\overleftrightarrow{\mathfrak{v}}
$$

We see, on the other hand, that by passing of one reactant over into the resultant the Zs of the assembly is multiplied by a factor $p^{\delta^{R}} / p^{\delta^{L}}$, inasmuch as the intermediate product of the steady reaction changes meanwhile hardly in concentration ${ }^{*}$. The multiplication being, however, over-all result of elementary reaction involved, it equals the multiplication due to the rate-determining step $p^{\delta^{F^{*}}} / p^{\delta^{I}}$, raised to $\mu$-th power i.e.

$$
p^{\hat{o}^{R}} / p^{\delta^{L}}=\left(p^{\delta^{\sigma}} / p^{\delta}\right)^{\bar{\nu}},
$$

since Zs remains unaffected because of the equilibrium relation Eq. (6.1) by any othar elementary reaction than the rate-determining step according to $\$ 22$, (ii).

The argument $p^{\delta^{F}} / p^{\delta^{I}}$ on the right hand side of Eq. (26.3) equals $\vec{v} / \vec{v}$ as it follows immediately from Eqs. $(24.1 . \vec{v})$ and $(24.1 . \stackrel{\leftarrow}{v})$, i.e.

$$
\frac{\vec{v}}{\stackrel{t}{v}}=\frac{p^{\delta^{F}}}{p^{\delta^{I}}}
$$

Eliminating now $p^{\delta^{F}} / p^{\delta^{I}}, \vec{v}, \overleftarrow{v}$ and $\overleftarrow{\mathfrak{v}}$ from Eqs. (26.1. $),(26.1 . \overline{\mathfrak{v}}),(26.2)$, (26.3) and (26.4), we have,

(*) Cf. $\$ 22$. 


$$
\vec{r}=\overrightarrow{\mathfrak{v}}\left\{1-\left(p^{\delta^{L}} / p^{\hat{o}^{R}}\right)^{\frac{1}{\mu}}\right\}
$$

or differentiating with respect to,

$$
\begin{gathered}
x=p^{\delta^{L}} / p^{\delta^{R}}, \\
\frac{\partial \vec{r}}{\partial x}=\frac{\partial \overrightarrow{\mathfrak{v}}}{\partial x}\left(1-x^{\frac{1}{\vec{x}}}\right)-\frac{1}{\mu} \overrightarrow{\mathfrak{v}} x^{\frac{1}{\mu}-1}
\end{gathered}
$$

or at equilibrium of the reaction when $x=1$,

$$
\frac{1}{\mu}=-\frac{1}{\vec{v}_{E q}}\left(\frac{\partial \vec{r}}{\partial x}\right)_{E q}
$$

or according to Eqs. (26.6) and (8.12),

$$
\frac{1}{\mu}=\frac{\dot{R T}}{\overrightarrow{\mathrm{v}}_{E q}}\left(\frac{\partial \vec{r}}{\partial\left(\mu^{\delta^{L}}-\mu^{\delta^{R}}\right)}\right)_{E_{q}}
$$

where the suffix Eq. signifies the values at equilibrium.

The number $\mu$ is expressed in a slightly different form by differentiating $\vec{r}$ with respect to $p^{\delta_{l}^{L}}$ : the result is, according to Eqs. (26.5). (26.6) and (25.5.L),

$$
\frac{\partial \vec{r}}{\partial p^{\delta_{l}^{L}}}=\frac{\partial \overrightarrow{\mathfrak{v}}}{\partial p^{\delta_{l}^{L}}}\left(1-x^{\frac{1}{\mu}}\right)-\overrightarrow{\mathfrak{b}} \frac{\nu_{l}^{L}}{\mu} x^{\frac{1}{\mu}} / p^{\delta_{l}^{L}}
$$

or at equilibrium

$$
\frac{\nu_{l}^{L}}{\mu}=-\frac{1}{\overrightarrow{\mathfrak{v}}_{E q}}\left(\frac{\partial \vec{r}}{\partial \log p^{\delta_{l}^{L}}}\right)_{E q}
$$

or by Eq. (8.12),

$$
\frac{\nu_{l}^{L}}{\mu}=\frac{R T}{\overrightarrow{\mathfrak{v}}_{E Q}}\left(\frac{\partial \vec{r}}{\partial \mu \delta_{l}^{L}}\right)_{E q}
$$

Eq. (26.8) or (26.10) allows us to determine $\mu$ experimentally provided $\vec{v}_{E q}$ is observed for instance by a proper use of isotopes. 
A Method of Statistical Mechanical Treatment of Equilibrium and Chemical Reactions.

\section{\$27. The Stoichiometrical Number of the Hydrogen Electrode Process.}

- The fitting of an alternative mechanism to the observed value of $\mu$ is exemplified below with the hydrogen electrode process.

If the hydrogen electrode reaction written down in the form,

has the mechanism,

$$
H_{2}=2 H^{+}+2 \varepsilon
$$

$$
H_{2} \rightarrow 2 H, \quad H A H^{*}+\epsilon
$$

i.e. if $H_{2}$ splits first into adsorbed hydrogen atoms and then each atom dissociates further into a hydrogen ion $\mathrm{H}^{+}$and a metal electron $\varepsilon$ of the electrode and the latter step is the rate-determining, $\mu$ is 2 , since then the step should take place twice as frequently as the total reaction.

If on the other hand, the mechanism is such that,

$$
H_{2} \uparrow H_{2}^{+}+\varepsilon, \quad H_{2}^{+} \rightarrow 2 H^{+}+\varepsilon
$$

or that $H_{2}$ splits first into the hydrogen molecule ion $\mathrm{H}_{2}^{+}$and a metal electron determining the rate, and $H_{2}^{+}$then further into $2 H^{+}$and $\varepsilon, \mu$ must be 1 .

The experimental determination of $\mu$ in the case of the hydrogen electrode process on platinum has been carried out by Horiuti and Ikusima ${ }^{(1)}$. The resultant current $\overrightarrow{\mathfrak{b}}_{E q}$ in Eq. (26.8) was measured by the electrode current, while $\overrightarrow{\mathbf{v}}_{E_{q}}$ by the rate of the exchange reaction between the hydrogen gas and the solution around the electrode ${ }^{(2)}$ and $p^{\hat{\sigma}^{L}} / p^{\hat{\sigma}^{R}}$ by the relation,

$$
-R T \log p^{\hat{o}^{L}} / p^{\hat{o}^{R}}=2 F \eta^{(*)}
$$

(1) Horiuti and Ikusima; Proc. Imp. Acad. Tokio, 15 (1939), 39.

(2) Horiuti and Polanyi; Nature, $132(1933), 819$.

Horiuti and Hirota; Sc. Pap. I.P.C.R. Tokio, 30 (1936), 151.

(*) Eq. (27.4) is derived as follows. For the reversible hydrogen electroce we have the equilibrium relation according to Eq. (6.1), i.e.

$$
p^{\prime} H_{2}=\left(p H^{+}\right)^{\circ}\left(p_{0}^{\varepsilon}\right)^{\circ}
$$

where $p_{0}^{\varepsilon}$ is the $p^{\delta}$ of the metal electron there. But since the reversible work of trangfering $-F$ electricity from the reversible electrode into the electrode in question is $-F y$ by definition, we have

$$
-R T \log p^{\varepsilon} / p_{0}^{\varepsilon}=-F \eta
$$

where $p^{\mathfrak{z}}$ is that of the metal electron in the electrode. But since according to Eq. (27.1), $p^{\delta^{L}}=p^{H_{2}}$ and $p^{\delta^{R}}=\left(p^{H^{+}}\right)^{2}\left(p^{\varepsilon}\right)^{2}$, we have, $p^{\delta^{L} /} / p^{\delta^{R}}=\left(p_{0}^{\varepsilon} / p^{\mathcal{Q}}\right)^{*}$ and hence Eq. (27.4). 
where $F$ signifies Faraday and $\eta$ the overvoltage of the electrode ${ }^{(*)}$. We obtain from Eqs. (26.6), (26.7) and (27.4) noting that $p^{\delta^{L}}=p^{\delta^{R}}$ at equilibrium and hence $\eta=0$, an expression,

$$
\frac{1}{\mu}=\underset{2 y_{\eta=\theta}}{R T}\left(\frac{\partial \vec{i}}{\partial \eta}\right)_{r_{i}=n}
$$

where $\vec{\psi}_{\eta=0}$ and $\vec{i}$ are currents corresponding respectively to rates $\vec{v}_{E_{\alpha}}$ and $\vec{r}$.

Horiuti and Ikusima ${ }^{(1)}$ thus found that $\mu=1$ for the hydrogen electrode process on platinum. The mechanism of Eq. (27.3) among the two alternatives advanced thus only fits the observed value of $\mu$.

\section{CHAPTER VI}

\section{APPLICATION-IV. HOMOGENEOUS REACTION}

$\$ 28$. The Rate of the Homogeneous Elementary Reaction.

We define $k^{\mathrm{x}}$ of the homogeneous elementary reaction in accordance with Eqs. (24.8. $),(24.2 . I)$ and (5.5) as that,

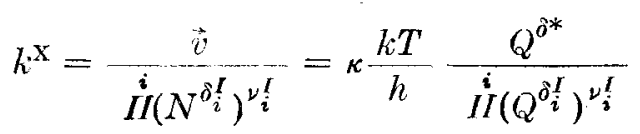

where suffix $\mathrm{x}$ signifies, $\sum_{i} \nu_{i}^{I}$ or the total number of molecules of the initial complex. $k^{\mathrm{x}}$ is the "rate constant" as called usually but not necessarily a constant inasumuch as $Q^{\delta}$ 's in Eq. (28.1) may vary if $\delta^{\prime} s$ are not ideal.

Denoting the quantities at infinite dilution of variable components by suffix 0 , we have,

$$
k^{\mathrm{x}}=\frac{\kappa}{\kappa_{0}} k_{0}^{\mathrm{x}} \frac{\stackrel{i}{I}\left(f^{\delta_{i}^{I}}\right)^{\nu_{i}^{I}}}{f^{\delta^{*}}}
$$

(*) The overvoltage is defined as the electrode potential referred to any reversible hydrogen electrode in the same solution of the same hydrogen concentration as that in the direct neighbourhood of the electrode in question.

(1) Horiuti and Ikusima; Proc. Imp. Acad. Tokio 15 (1939), 39. 
where

$$
k_{0}^{\mathrm{X}}=\kappa_{0} \frac{k T}{h} \frac{Q_{0}^{\delta^{*}}}{\underset{I I}{i}\left(Q_{0}^{\delta^{I}}\right)^{\nu I}}, \quad f^{\hat{o}}=\frac{Q_{0}^{\delta}}{Q^{\delta}}
$$

$f^{\delta}$ may be identified with activity coefficient inasmuch as it becomes unity at the infinite dilution and bears the relation to $\mu^{\delta}$ according to Eqs. (28.4), (14.5) and (14.6) that,

$$
\mu^{\delta}=\mu_{1,0}^{\delta}+R T \log f^{\delta} N^{\delta}, \text { where } \mu_{1,0}^{\delta}=-R T \log Q_{0}^{\delta}
$$

The relation of the form of Eq. (28.5) was advanced by Brönsted(1) and Wynne-Jones and Eyring(2) by analogy to thermodynamies regarding the activated complex as thermodynamical chemical species without defining $f^{\delta}$ on the statistical mechanical basis.

In the case of a gas $k_{0}^{\mathrm{X}}$ is independent of the pressure, $Q_{0}^{\delta}$ 's being equal to that in vacuum. $Q_{0}^{\delta^{*}}$ is hence calculable with reference to a single reaction complex in vacuum.

\$29. The Rate Constant due to Eyring, Evans and Polanyi.

Eq. (28.1) or (28.3) is identical in the form with those obtained by Eyring ${ }^{(5)}$, Evans and Polanyi ${ }^{(4)}$ but not quite in its implication. The latter authors derive the identical form of Eq. (28.3) by defining the activated complex $\delta_{E}^{*}(g)$ arbitrarilly with reference to the saddle point on the potential energy surface of an isolated $\bar{\delta}$ and by assuming the chemical equilibrium between the initial and the activated complex or by expressing the concentration $N^{\delta_{E}^{*}(g)}$ of the latter according to the equilibrium relation $\mathrm{Eq}$. (16.1) as that,

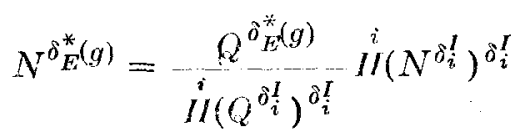

Multiplying the latter by the "universal frequency" $k T / h$, the frequency of transition through the saddle point in the forward direction for $\delta_{E}^{*}(g)$ and by the chance $k$ "that having once crossed the barrier, the activated

(1) Brönsted; Z. physik, Chem. 102 (1922), 109.115 (1925), 337.

(2) Wynne-Jones and Eyring; J. Chem. Phys. 3 (1935), 492.

(3) Eyring; J. Chem. Phys. 3 (1935), 107.

(4) Evans and Polanyi ; Trans. Faraday Soc. 31 (1935), 875. 
complex does not return"(1), they arrive at the rate expression and hence at the identical form of Fq. (28.1). Extending now the latter form by analogy, they go back to that of Eq. (28.2) for the elementary reaction in liquid, $Q^{\delta *}$ there being, according to them, that of quasi-gas molecule moving in the mean potential field of the enviroment.

It is implied in the theory of Eyring ${ }^{(2)}$, Evans and Polanyi ${ }^{(3)}$ that the saddle point is at least approximately narrowest pass on the path from the initial to the final state. This may be practically true in many cases but associated with difficulty in some cases.

Eyring assumes, for example, in accounting for the negative temperature coefficient of the elementary reaction,

$$
2 \mathrm{NO}+\mathrm{O}_{2} \rightarrow 2 \mathrm{NO}_{2}(2)
$$

that the activated complex consisting of the three molecules $2 N O$ and $\mathrm{O}_{2}$ has the same minimum potential energy as the initial complex, which corresponds to the lowest possible saddle point. As pointed out by O.K. Rice $^{(4)}$ however the temperature coefficient cannot be zero or negative if such saddle point is even approximately narrowest to pass through. This difficulty is of course not associated with the present method where the critical complex is originally defined with referrence to the narrowest configuration itself.

Another difficulty with their method is due to the assumption of the equilibrium relation between the initial and the activated complex. We consider first the state of equilibrium between the initial and the final complex and then that of the irreversible state brought about from the former by removing the final complex. If the transmission coefficient is unity or the reacting complex. which transits the state of the activated complex forward, originate all in the initial complex, the rate of the transition remains of course unaffected by removing of the final complex. The equilibrium relation is then applicable in this sense which is assumed by Wynne-Jones and Eyring( ${ }^{(\varepsilon)}$ for general validity.

The assumption is not, however, legitimate even in this sense when

(1) Eyring; Trans. Faralay Soc: 34 (1988), 41.

(2) Eyring; J. Am. Chem. Soc. 57 (1935), 985.

(3) Evans and Polanyi ; Trans. Paraday Soc. 31 (1935), 875.

(4) O. K. Rice; J. Chem. Phys. 4 (1946), 53.

(5) Wynne-Jones and Eyring; J. Chem. Phys. 3 (1935), 492. 
A Method of Statistical Mechanical Treatment of Equilibrium and Chemical Renctions.

the transmission coefficient is appreciably less than unity, or, when only a small fraction of the reaction complexes once traversed the activated state completes the elementary reaction ${ }^{(*)}$. Admitting with Eyring, Evans and Polanyi that the activated state is the narrowest pass throughout the course from the initial to the final state, we may conclude that at equilibrium the activated complex transiting in either direction will equally likely reach the initial as well as the final state, and any one there is equally likely having come from the either state. By removing now the final complex in this case, the activated complex, which originating in the final complex transits the activated state forward, falls off, the total number of activated complexes transiting forward being thus almost halved. The equilibrium relation is thus inapplicable here even in the above sense.

This latter difficulty is, however, only formal, since the final rate expression Eqs. (28.1) or (28.3), of the identical form is derived according to the present method without resorting to the equilibrium relation.

It seems to the present author that Fowler(1), Guggenheim and Weiss $^{(2)}$ criticises the method of Eyring ${ }^{(3)}$, Evans and Polanyi ${ }^{(4)}$ because of the equilibrium relation with the conception of the latter case or that of the diffusion-type as called by the present author ${ }^{(5)}$ whereas Fyring, Evans and Polanyi( ${ }^{(6)}$ defend with the conception of the former case or that of the effusion type. 'They both are justified so far as the respective extreme case is concerned.

Rabinowitch ${ }^{(7)}$ Christiansen $^{(8)}$ and Norrish ${ }^{(9)}$ follow a different line of developing the theory of the elementary reaction from that of Eyring(3), Evans and Polanyi ${ }^{(4)}$ with special reference to the diffusion type. The

(*) We consider here only elementary reactions which proceeds adiabatically, ef. $\$ 23$.

(1) Fowler; Trans. Faraday Soc, 34 (1938), 75, 124.

(2) Guggenheim and Weiss; Trans. Faraday Soc. 34 (1938), 57.

(3) Eyring; J. Chem. Phys. 3 (1935). 107.

(4) Evans and Polanyi ; 'Trans. Faraday Soc., 31 (1935), 875.

(5) Horiuti ; Bull. Chem. Soc. Japan, Katayama Commemoration Vol. 13 (1938), 20.

Sc. Pap. I.P.C.R. Tokio, 34 (1938), 1174.'

(6) Cf. Faraday Society, General Discussion "Reaction Kineties" Trans. Faraday Soc. 34 (1938), 1, 265.

(7) Rabinowitch; Trans. Faraday Soc. 34 (1938), 113.

(8) Christiansen; Trans Faraday Soc. 34 (1938), 73; J. Chem. Phys. 7 (1939), 653.

(9) Norrish ; Trans Faraday Soc. 34 (1938), 73. 
procedure of the former group of authors seems, however, to be looked upon by Eyring as an alternative manner of describing the unique reality ${ }^{*}$ rather than as dealing, as actually they are, with the other extremity of the reality.

Experimental meterials hitherto found unfortunately fit the picture of the effusion type, or equally well the both. It is desirable to find examples which, fitting exclusively that of the diffusion type, lead us innevitably to the correct synthesis of these antithesis.

\section{$\$ 30$. The Thermodynamical Form of the Homogeneous Rate.}

In this section the rate and the rate constant will be expressed in thermodynamical terminology: it is however neither claimed nor needs to be claimed thereby that the critical complex behaves like a thermodynamical ehemical species or that recognized directly by thermodynamical (stoichiometrical) procedure; every quantity and relation here dealt with are purely of the statistical mechanical formulation being clad in thermodynamical terms.

Eq. (24.1. $\vec{v})$ for the rate of the elementary reaction is expressed by substituting $p^{\delta}$ 's formally from Eq. (8.12) with due regard to Eqs. $\left(24.2 . \delta^{I}\right),(13.21 . \mu)$ and $(13.22 . \mu)$ as follows,

$$
\vec{v}=\kappa \frac{k T}{h} e^{-\frac{\Delta \|^{*} F_{V}}{R T}}=\kappa \frac{k T}{h} e^{-\frac{\Delta \Delta^{*} Z_{P}}{R T}}
$$

where

$$
d^{*} F_{V}=\breve{F}_{V}^{\delta^{*}}-\sum_{i} \nu_{i}^{I} \bar{F}^{\delta_{i}^{I}}=U^{*} Z_{P}=Z_{P}^{\delta^{*}}-\sum_{i} \nu_{i}^{I} \bar{Z}^{\delta_{i}^{I}}
$$

Denoting the critical increment of any extensive property by prefix $4^{*}$ and the associated condition of constant volume or of constant pressure respectively by suffix $V$ or $P$ as above we have from Eqs. (13.21. $\mu$ ), $(13.22 . \mu)$ and $(13.23 . \mu)$ that,

$$
\Delta !^{*} F_{V}=\Delta \Delta^{*} Z_{P}=\Delta \Delta^{*} U_{V}-T \Delta^{*} S_{V}=\Delta^{*} X_{P}-T \Delta \Delta^{*} S_{P}
$$

and hence Eq. (30.1) in the form,

(*) Cf. Eyring's comment on the work of Christiansen; J. Chem. Phys. 7 (1939), 654. 
A Method of Statistical Mechanical Treatment of Equilibrium and Chemical Reactions.

$$
\vec{v}=\kappa \frac{k T}{h} e^{\frac{\|^{*} S_{V}}{R}} e^{-\frac{\Delta^{*} U_{V}}{R T}}=\kappa \frac{k T}{h} e^{\frac{d^{*} S_{P}}{R}} e^{-\frac{\|^{*} X_{P}}{R T}}
$$

Eq. (28.1) for $k^{x}$ is expressed according to Eqs. (14.6) and (14.17. $\mu$ ) in the form,

$$
k^{X}=\kappa \frac{k T}{h} e^{-\frac{\Delta^{*} F_{V, 1}}{R T}}=\kappa-\frac{k T}{h} e^{-\frac{\Delta^{*} Z_{P, 1}}{R T}}
$$

where

$$
\dot{\Delta}^{*} \vec{F}_{V, 1}=\bar{F}_{V, 1}^{\delta^{*}}-\sum_{i} \nu_{i}^{I} \bar{F}_{V, 1}^{\delta_{i}^{I}}=\Delta^{*} Z_{P, 1}=\bar{Z}_{P^{*}, 1}^{*}-\sum_{i} \nu_{i}^{I} Z_{P, 1}^{\delta_{i}^{I}}=\mu_{1}^{\delta^{*}}-\sum_{i} \nu_{i}^{I} \mu_{1}^{\delta_{i}^{I}}
$$

Defining increments $A^{*} U_{V, 1}, \Delta^{*} X_{P, 1}, \Delta_{i}^{*} S_{V, 1}$ and $A^{*} S_{P, 1}$ of the "standard state" similarly as $U^{*} F_{V, 1}$ or $\Delta^{*} Z_{P, 1}, k^{X}$ is further expressed according to Eqs. (14.21) in the form,

$$
k^{x}=\kappa \frac{k T}{h} e^{-\frac{\Delta^{*} U_{V, 1}-T \Delta^{*} S_{V, 1}}{R T}}=\kappa \frac{k T}{h} e^{-\frac{d^{*} X_{P, 1}-T \|^{*} S_{P, 1}}{R T}}
$$

where

$$
\Delta i^{*} F_{V, 1}=\left.\Delta\right|^{*} U_{V, 1}-\left.T \Delta\right|^{*} S_{V, 1}=\Delta{ }^{*} Z_{P, 1}=\Delta 1^{*} X_{P, 1}-\left.T \Delta\right|^{*} S_{P_{1},}
$$

Critical increaments and $k^{X}$ are constant at constant temperature and pressure provided that components of variable concentration are ideal and $\kappa, \alpha$ and $\beta$ remain respectively constant.

Interrelations among critical increments are readily derived from the relations among partial molal quantities developed in $\$ 13$ and $\$ 14$ as follows,

$$
\begin{aligned}
& \Delta{ }^{*} F_{V}=\left.\Delta\right|^{*} Z_{P}=\left.\Delta\right|^{*} F_{P}+P d^{*} V_{P} \\
& \Delta i^{*} S_{P}=u^{*} S_{V}+\frac{\alpha}{\beta} \Delta i^{*} V_{P} \\
& \Delta^{*} X_{P}=\Delta^{*} U_{V}+T \frac{\alpha}{\beta} \Delta^{*} V_{P}=\Delta \Lambda^{*} U_{P}+P \Delta \|^{*} V_{P} \\
& \left.\left(\frac{\partial \Delta^{*} F_{V}}{\partial T}\right)_{V}=-\Delta\right]^{*} S_{V}
\end{aligned}
$$




$$
\begin{aligned}
& \left(\frac{\partial \Delta^{*} Z_{P}}{\partial T}\right)_{P}=-U^{*} S_{P} \\
& \left(\frac{\partial U^{*} F_{V}}{\partial P}\right)_{T}=\left(\frac{\partial \Delta I^{*} Z_{P}}{\partial P}\right)_{T}=U^{*} V_{T} \quad(30.9 . F V) \\
& d^{* *} F_{V, 1}=d^{*} Z_{P, 1}=\Delta d^{*} F_{P, 1}+P d^{*} V_{F} \quad(30.10 . \mathrm{F}) \\
& J_{i}^{*} S_{P, 1}=J^{*} S_{V, 1}+\frac{\alpha}{\beta} \|^{*} V_{P} \\
& \left.J^{*} X_{P, 1}=A^{*} U_{V, 1}+T_{\beta}^{a}\right\rfloor^{*} V_{P}=J^{*} U_{P, 1}+P J^{*} V_{T} \\
& \left(\frac{\partial \Delta^{*} F_{V, 1}}{\partial T}\right)_{V}=-\Delta I^{*} S_{V, 1} \\
& \left(\frac{\partial \Delta^{*} Z_{P, 1}}{\partial T}\right)_{P}=-U^{*} S_{P, 1}+\nu^{*} R T_{r} \\
& \left(\frac{\partial \Delta \|^{*} F_{V, 1}}{\partial P}\right)_{T}=\left(\frac{\partial \Delta \|^{*} Z \dot{P}_{-1}}{\partial P}\right)_{T^{\prime}}=\Lambda^{*} V_{P}-\nu^{*} \operatorname{RT} \theta \quad(30.10 . \mathrm{FV}) \\
& \left(\frac{\partial d^{*} X_{P, 1}}{\partial P}\right)_{T}=\Lambda^{*} V_{P}-T\left(\frac{\partial d^{*} V_{P}^{*}}{\partial T}\right)_{P} \\
& \left.J^{*} F_{V, 1}=\right\rfloor^{*} F_{V}-F T \log N^{*}=\left.\Delta\right|^{*} Z_{P, 1}=\left.\Delta\right|^{*} Z_{P^{-}} R T \log \bar{N}^{*} \quad(30.12 . F) \\
& \Delta^{*} S_{V, 1}=\bigsqcup^{*} S_{V}+R \overline{\log } \bar{N}^{*} \\
& \Delta_{1}^{*} S_{P, 1}=J^{*} S_{P}+R \log \bar{N}^{*} \\
& U^{*} U_{V, 1}=U^{*} U_{V} \\
& \Delta^{*} X_{F, 1}=\|^{*} X_{P} \\
& \log N^{*}=\log N^{\partial^{*}}-\sum_{i} \nu_{i}^{I} \log N^{\hat{o}_{i}^{I}} \\
& 2^{*}=1-\sum_{i} \nu_{i}^{l} \text {. }
\end{aligned}
$$

In the special case of a gas when,

$$
-60-
$$


A Method of Statistical Mechanical Treatment of Equilibrium and Chemical Reactions.

$$
\|^{*} V_{P}=\nu^{*} \frac{R T}{P}, \alpha=\frac{1}{T} \text { and } \beta=\frac{1}{P}
$$

$(30.14 . V) .(30.14 . \alpha),(30.14 . \beta)$

Eqs. (30.9), (30.10) and (30.11) assume respectively the forms,

$$
\begin{aligned}
& \Delta \|^{*} F_{V}=\left.\Delta\right|^{*} Z_{P}=\left.\Delta\right|^{*} F_{P}+\nu^{*} R T \\
& \Delta !^{*} S_{P}=\Delta \|^{*} S_{V}+\nu^{*} R \\
& \Delta I^{*} X_{P}=\Delta^{*} U_{V}+\nu^{*} R T=\Delta I^{*} U_{P}+\nu^{*} R T \\
& \Delta)^{*} U_{V}=\left.\Delta\right|^{*} U_{P}=\Delta I^{*} U \\
& \left(\frac{\partial \Delta_{i}^{*} F_{V}}{\partial T}\right)_{V}=-\Delta^{*} S_{V},\left(\frac{\partial \Delta \|^{*} Z_{P}}{\partial T}\right)_{P^{\prime}}=-\left.\Delta\right|^{*} S_{P},\left(\frac{\partial \Delta^{*} \dot{F} V}{\partial P}\right)_{T}=\left(\frac{\partial \Delta \|^{*} Z_{P}}{\partial P}\right)_{T}=\nu^{*} \frac{R T^{*}}{P} \\
& \text { (30.15.FS), (30.15.ZS), (30.15.FV) } \\
& \left.\left.\Delta\right|^{*} F_{V, 1}=\Delta\right)^{*} Z_{P, 1}=\Delta{ }^{*} F_{P, 1}+\nu^{*} R T \\
& A I^{*} S_{V, 1}=\Delta I^{*} Z_{P, 1}+\nu^{*} R \\
& \left\|^{*} X_{P, 1}=\left.\Delta\right|^{*} U_{V, 1}+\nu^{*} R T=\Delta\right\|^{*} U_{P, 1}+\nu^{*} R T,\left.\quad \Delta\right|^{*} U_{V, 1}=\left.\Delta\right|^{*} U_{P, 1} \\
& (30.16 . \mathrm{X}),(30.16 . \mathrm{U}) \\
& \left(\frac{\partial \Delta \|^{*} F_{V, 1}^{\prime}}{\partial T}\right)_{V}=-\Delta I^{*} S_{V, 1} \\
& \left(\frac{\partial \Delta \|^{*} Z_{P, 1}}{\partial T}\right)_{P}=-\left.\Delta\right|^{*} S_{P, 1}+2^{*} R \\
& \left(\frac{\left.\partial \Delta\right|^{*} F_{V, 1}}{\partial P}\right)_{T}=\left(\frac{\left.\partial d\right|^{*} Z_{P, 1}}{\partial P}\right)_{T^{*}}=0 \\
& \left(\frac{\partial \Delta l^{*} X_{P, 1}}{\partial P}\right)_{T}=0
\end{aligned}
$$

whereas Eq. (30.12) remains intact.

\$31. The Temperature Variation of the Rate and the Rate Constant.

Our treatment for the variation of the rate with temperature will be restricted to the elementary reaction of the effusion type ${ }^{(*)}$ for which

(*) See p. 57. Cf. Horiuti; Bull. Chem. Soc. Japan, Katayama Commemoration Vol. 13 (1938), 20. Sc. Pap. I.P.C.R. Tokio, 34 (1938), 1174. 
$\kappa$ is constantly unity. The temperature variation of the rate $\vec{v}$ is readily expressed according to $\mathrm{Eq} .(30.1)$ in the form,

$$
R T^{2}\left(\frac{\partial \log \vec{v}}{\partial T}\right)_{P}=R T+\Delta \|^{*} Z_{P}-T\left(\frac{\partial \Delta \|^{*} Z_{P}}{\partial T}\right)_{P}
$$

and

$$
R T^{2}\left(\frac{\partial \log \vec{v}}{\partial T}\right)_{V}=R T+\Delta I^{*} F_{V}-T\left(\frac{\partial \Delta^{*} F V}{\partial T}\right)_{V}
$$

and hence according to Eqs. (30.3) and (30.9),

$$
\begin{aligned}
& R T^{2}\left(\frac{\partial \log \vec{v}}{R T}\right)_{P}=R T+\left.\Delta\right|^{*} X_{P} \\
& R T^{2}\left(\frac{\partial \log \vec{v}}{R T}\right)_{V}=R T+\left.\Delta\right|^{*} U_{V}
\end{aligned}
$$

The temperature variation of the rate constant of the elementary reaction is expressed in the form of the Arrhenius activation energy by differentiation of Eq. (30.5), i.e:-

$$
\begin{aligned}
& R T^{2}\left(\frac{\partial \log k^{X}}{\partial T}\right)_{P}=R T+\left.\Delta\right|^{*} Z_{P_{, 1}}-T\left(\frac{\partial \Delta^{*} Z_{P_{, 1}}}{\partial T}\right)_{P} \\
& R T^{2}\left(\frac{\partial \log k^{X}}{\partial T}\right)_{V}=R T+\left.\Delta\right|^{*} F_{V, 1}-T\left(\frac{\left.\partial \Delta\right|^{*} F_{V, 1}}{\partial T}\right)_{V}
\end{aligned}
$$

and according Eqs. (30.8) and (30.10)

$$
\begin{aligned}
& R T^{2}\left(\frac{\partial \log k^{x}}{\partial T}\right)_{P}=R T+\Delta A^{*} X_{P}-\nu^{*} R T^{2} \alpha \\
& R T^{2}\left(\frac{\partial \log k^{X}}{\partial T}\right)_{V}=R T+\left.\Delta\right|^{*} U_{V}
\end{aligned}
$$

The difference between the above two forms of the temperature variation is expressed according to Eq. (30.9.X) as that,

$$
R T^{2}\left(\frac{\partial \log k^{X}}{\partial T}\right)_{P}-R T^{2^{\prime}}\left(\frac{\partial \log k^{X}}{\partial T}\right)_{V}=\left.T \frac{\alpha}{\beta} \Delta\right|^{*} V_{P}-\nu^{*} R T^{2} \alpha
$$


A Method of Statistieal Mechanical Treatment of Equilibriam and Chemical Reactions.

Assuring that $T=300 \mathrm{~K}, \mathrm{U}^{*} V_{P}=100 \mathrm{cc}, \alpha=10^{-3} \mathrm{deg}^{-1}, \beta=10^{-4} \mathrm{~atm}^{-1}$ and $\nu^{*}=-1$ which corresponds to the ordinary case of the bimolecular elementary reaction in liquid, we find $7 \mathrm{Kcal}$ for the difference which is by no ineans negligible.

The expression for the difference is derived by Evans and Polenyi(1) raising the importance of the "Arrhenius activation energy at constant volume" $R T^{2}\left(\frac{\partial \log k^{x}}{\partial T}\right)$ for elucidation of the reaction mechanim in liquid who, however, leave out the second term $\nu^{*} R T^{2}{ }_{i \alpha}$ in the above equation.

The latter term, although minor in the above example of the liquid assembly, becomes so large in gas that it just cancels the first according to Eqs. (30.14). Both the "activation energies" are now identical with each other being given as that,

$$
R T \cdot\left(\frac{\partial \log k^{x}}{\partial T}\right)_{P}=R T^{2}\left(\frac{\partial \log k^{X}}{\partial T}\right)_{V}=R T+\Delta^{*} U
$$

where

$$
\Delta^{*} U=\Delta{ }^{*} U_{P}=\Delta^{*} U_{V}
$$

\$32. Pressure Variation of the Rate.

Our treatment of the pressure variation is restricted as noted in the foregoing section to the case of effrion type. We have readily according to Eqs. (30.1) and (30.9.FV) tiat,

$$
\left(\frac{\partial \log \vec{v}}{\partial P}\right)_{T}=\frac{\Delta I^{*} V_{P}}{\vec{R}^{T}}
$$

Similar expression for $k_{0}^{x}$ is obtained fom Eqs. (30.5) and $(30.10 . F V)$ as that,

$$
\left(\frac{\partial \log k^{x}}{\partial P}\right)_{T}=-\frac{L^{*} V_{P}}{R T}+\beta \nu^{*}
$$

Differentiating Eq. (31.8.P) with respect to $P$ with regard to $q$. (30.11), or $\mathrm{Eq}$. (32.2) with respect to $T$, we have,

(1) Eva:1s and Polanyi ; Trans. Faraday Soc. 31 (1935), 875. 


$$
\frac{\partial^{2} \log k^{x}}{\partial T \cdot \partial P}=\frac{\Delta l^{*} V_{P}}{R T^{2}}-\frac{1}{R T}\left(\frac{\partial \Delta l^{*} V_{P}}{\partial T}\right)_{P}+\nu *\left(\frac{\partial \beta}{\partial T}\right)_{P}
$$

or

$$
\left(\frac{\partial E_{P}}{\partial P}\right)_{Y}=\Delta \|^{*} V_{P}\left\{1-T\left(\frac{\partial \log \Delta \|^{*} V_{P}}{\partial T}\right)_{P}\right\}-\nu^{*} R T^{2}\left(\frac{\partial \alpha}{\partial P}\right)_{T}
$$

where

$$
E_{P}=R T^{2}\left(\frac{\partial \log k^{X}}{\partial T}\right)_{P}
$$

and

$$
\left(\frac{\partial \alpha}{\partial P}\right)_{T}=\frac{\partial^{2} \log V}{\partial P \cdot \partial T}=-\left(\frac{\partial \beta}{\partial T}\right)_{P}
$$

Evans and Polanyi(1) and Guggenheim ${ }^{(2)}$ derived respectively the similar expression for $\left(\frac{\partial E_{P}}{\partial P}\right)_{T}$, the former authors, however, leaving out the term $\nu^{*} R T^{\dot{2}}\left(\frac{\partial x}{\partial P}\right)_{T}$, whereas the latter including a term valid in the special case of the bimolecular association when $\nu^{*}=-1$.

\section{\$33. Comment on the Controversies on the Thermodynamical Treatment} of the Rate.

As detailed in the foregoing section due distinction must be made, for exact thermodynamical treatment of the rate, between the partial molal quantities and the total quantities on the one hand, and, between the critical increments at constant pressure and those at constant volume on the other hand except in the case of the gas. Such distinction is of course not merely the matter of the thermodynamical treatment of the rate, but might be directed back to the pure thermodynamical treatment of the equilibrium.

Less precaution seems to have hitherto been paid on this respect, as̃ might naturally be expected from the history of the theory of the rate developed in analogy to the thermodynamical theory of equilibrium in a gas. The situation seems, however, to have resulted in unfortunate condusions and controversies, which are going to be analysed and com-

(1) Evans and Polanyi ; Trans. Faraday Soc. 32 (1936), 1333.

(2) Guggenheim; Trans. Faraday Soc. 33 (1937), 697. 
mented upon hereafter in the light of the present thermodynamical theory of the rate.

Moelwyn-Hughes ${ }^{(1)}$ puts forward the following expression for the dependence of the Arrhenius activation energy $E_{A}$ on hydrostatical pressure $I^{\prime}$.

$$
E_{A}=\left.\Delta\right|^{*} E+\left.P \Delta\right|^{*} V\left\{1-T\left(\frac{\left.\partial \log \Delta\right|^{*} V}{\partial T^{\prime}}\right)_{P}-T\left(\frac{\partial \log P}{\partial T}\right)_{V}\right\}
$$

where $\Delta^{*} E$ or $\Delta \|^{*} V$ is, as he states, the increase in internal energy or volume associated with the change from ordinary to activated molecules.

Fvans and Polanyi ${ }^{(2)}$ advance another expression,

$$
E_{A}=E_{0}+\left.P \Delta\right|^{*} V\left\{1-T\left(\frac{\left.\partial \log \Delta\right|^{*} V}{\partial T}\right)_{P}\right\}
$$

for the relation, where $E_{0}$ is $E_{A}$ at $P=0$. The latter expression may be derived along the line of procedure of Evans and Polanyi by neglecting $R T^{2} \nu^{*}\left(\frac{\partial \alpha}{\partial P}\right)_{T}$, identifying $E_{A}$ to $E_{P}$ in Eq. (32.3) and integrating the latter with respect $P$, regarding $\Delta \|^{*} V\left\{1-T\left(\frac{\partial \log \Delta^{*} V}{\partial T}\right)_{P}\right\}$ constant. Evans and Polanyi( ${ }^{(3)}$ claim Moelwyn-Hughes' Eq. (33.1) contradicts thermodynamics, because the latter is inconsistent, as they prove, with their Eq. (33.2).

Moelwyn-fiughes starts from the assumption that,

$$
k_{0}^{X}=\operatorname{const} e^{-\frac{\Delta^{*} \varphi}{R T}}
$$

and that

$$
\left.\Delta\left\|^{*} \phi=\right\|\right|^{*} E+P\|\|^{*} V-\left.T \Delta\right|^{*} S
$$

where $\|^{*} \boldsymbol{p}$ is, as he states, the critical increment of the (Helmholtz's) free energy. By differentiation of $\mathrm{Eq}$. (33.3) he arrives at $\mathrm{Eq}$. (33.1).

In oder to follow his line of reasoning exactly the critical increments involved in the assumption, $\left.\Delta\right|^{*} \phi,\left.\Delta\right|^{*} E,\left.\Delta\right|^{*} S^{*}$ and $\left.\Delta\right|^{*} V$ have to be distinguished with respect to their associated conditions. For being the incre-

\footnotetext{
(1) Moelwyn-Hughes; Trans. Faraday Soc. 32 (1936), 1723.

(2) Evans and Polanyi; Trans. Faraday Soc. 32 (1936), 1333.

(3) Evans and Polanyi; Trans. Faraday Soc. 33 (1937), 448.
} 
ment of the Helmholtz's free energy, $\left.\Delta\right|^{*} \boldsymbol{\rho}$ must be any one of $\Delta^{*} F_{V, 1}$, $\Delta \|^{*} F_{P, 1},\left.\Delta\right|^{*} F_{V}$ and $\left.\Delta\right|^{*} F_{P}$. It must be however either $\left.\Delta\right|^{*} F_{V, 1}$ or $\Delta \|^{*} F_{P_{1},}$ rather than $\left.\Delta\right|^{*} F_{V}$ or $\left.\Delta\right|^{*} F_{P}$ since otherwise, $k_{0}^{X}$ depends necessarily on the concentrations of the initial complex. The $\Delta \|^{*} F_{V, 1}$ is expressed according to Eqs. (30.8) and (30.10.X) as that,

$$
\left.\Delta\right|^{*} F_{V, 1}=\left.\Delta\right|^{*} U_{P, 1}+\left.P \Delta\right|^{*} V_{P}-T \Delta \|^{*} S_{P, 1}
$$

whereas $\left.\Delta\right|^{*} F_{P, 1}$ according to Eq. $(30.10 . F)$ as that,

$$
\left.\Delta\right|^{*} F_{P, 1}=\left.\Delta\right|^{*} U_{P, 1}-\left.T \Delta\right|^{*} S_{P, 1}
$$

We see that for $\left.\Delta\right|^{*} V_{p}$ to persist in the expression of $\left.\Delta\right|^{*} \phi$, the latter must be expressed by Eq. (33.5.V) which fixes the exact meaning of notations in Eq. (33.4). Moelwyn-Hughes' Eq. (33.1) appears now in the form,

$$
E_{A}=\left.\Delta\right|^{*} U_{P, 1}+\left.P \Delta\right|^{*} V_{P}\left\{1-T\left(\frac{\left.\partial \log \Delta\right|^{*} V_{P}}{\partial T}\right)_{P}-T\left(\frac{\partial \log P}{\partial T}\right)_{V}\right\}
$$

By Eq. (33.3), (33.5), (30.8) and (30.10) we have however, either,

$$
R T^{2}\left(\frac{\partial \log k_{0}^{X}}{\partial T}\right)_{P}=\left.\Delta\right|^{*} X_{P}-\nu^{*} R T^{2} \alpha
$$

or

$$
R T^{2}\left(\frac{\partial \log k_{0}^{X}}{\partial T}\right)_{V}^{*}=\left.\Delta\right|^{*} U_{V}
$$

Equating the former to the Arrhenius activation energy $E_{A}$, which is according to Evan and Polanyi( ${ }^{(1)}$ always to be referred to constant pressure, and transforming $\left.\Delta\right|^{*} X_{P}$ by Eq. $(30.10 . X)$, we have,

$$
E_{A}=E_{P}=\Delta I^{*} U_{P, 1}+\left.P \Delta\right|^{*} V_{P}-\nu^{*} R T^{2} \alpha
$$

We see that differential coefficients $T\left(\frac{\partial \log \Delta \|^{*} V_{P}}{\partial T}\right)_{P}$ as well as $T\left(\frac{\partial \log P}{\partial T}\right)_{V}$ in Eq. (33.6) are here missing, the term $-\nu^{*} R T^{\mathbf{2} \alpha}$ appearing instead.

(1) Evans and Polanyi ; Trans. Faraday Soc. 33 (1937), 448. 
Evans and Polanyi, criticizing Moelwyn-Hughes' procedure of deriving his Eq. (33.1), state that the appearance of the third term $T\left(\frac{\partial \log P}{\partial T}\right)_{V}$ in the parenthesis is due to a mistake of differentiation and by performing the differentiation correctly an expression without the term $T\left(\frac{\partial \log P}{\partial T}\right)_{V}$ in coincidence with their Eq. (33.2) is obtained. But we see above that by carrying out the differentiation more correctly even the term $T\left(\frac{\left.\partial \log \Delta\right|^{*} V_{P}}{\partial T}\right)_{P}$ disappears, which affords a strong feature of resemblance of Eq. (33.1). with (33.2). The erronous term $T\left(\frac{\partial \log \Delta \|^{*} V_{P}}{\partial T}\right)_{F}$ creeps into the final expression, if the differentiation of $-\frac{\Delta \|^{*} U_{P, 1}-\left.T \Delta\right|^{*} S_{P, 1}}{R T}$ with respect to $T$ is incorrectly taken to be $\frac{\Delta \|^{*} U_{P, 1}}{R T^{2}}$ by analogy to the differentiation of $-\frac{U-T S}{R T}$ at. constant volume, which gives exactly $\frac{U}{R T^{2}}$ according to Eqs. (8.8) and (8.10) or by analogy to the differentiation of $-\frac{\Delta 1^{*} U_{V .1}-\left.T A\right|^{*} S_{V .1}}{R T}$ at constant volume resulting exactly in $\frac{\Delta \|^{*} U_{V, 1}}{R T}$ according to Eqs. (30.8) and (30.10.FS).

It may be seen that the neglection of the distinction of the partial molal quantity from the total quantity and of the associated condition of the critical increment is quite misleading.

Guggenheim(1) developed the thermodynamical theory of the rate correctly, arriving at the identical forms of Eqs. (33.7.P) and (33.8) although the distinction between the' constant pressure and the constant volume is not explicitly noted or rather all partial molal quantities are referred to constant pressure.

$\mathrm{He}$ starts thereby from the definition of the ideally dilute solution with the independent variables of temperature, pressure and mol fraction, giving quite different formulations for the thermodynamics in gas from. that in liquid, whereas here the ideal solution is defined with the homogeneous fluid in accordance with Guggenheim's particular form in liquid, the other in gas being automatically derived as its special case. We

(1) Guggenheim; Trans. Faraday Soc. 33 (1937), 607. 
note that the term $\nu^{*} R T^{2} \alpha$, in Eqs. (30.10.ZS), (31.8.P) and (31.9), taken into account with its particular value by Guggenheim, takes care, as shown in $\$ 30$ and $\$ 31$, with proper precaution on the condition of partial molal quantities, to include the thermodynamics of the elementary reaction in gas as the special case.

On commenting on the papers of Moelwyn-Hughes and of Evans and Polanyi on the basis of his theory, Guggenheim(1) objects Evans and Polanyi's view that $k_{0}^{x}$ should be differentiated at constant pressure on the ground that measured values of $E_{A}$ at ordinary pressures as well as at hydrostatical pressure are all referred to constant pressure ${ }^{(2)}$, stating that "the correct reason of keeping the pressure constant, is that temperature, pressure and mol fractions are the independent variables in the simple formulæ by which ideal systems have been defined and there- fore also in all formula derived therefrom."(1). We see no reason why we should to be faithful to this set of variables throughout: with proper transformations and with proper specifications of associated conditions, as shown in foregoing sections, we arrive at even simpler form of the temperature variation of $k_{0}^{X}$ at constant volume. We quite agree with Evans and Polanyi's view of referring measured $E_{A}$ to constant pressure and of raising the importance of that at constant volume derivable therefrom for the theory of reaction in solution.

\section{CHAPTER VII}

\section{APPLICATION-V. HETEROGENEOUS REACTIONS.}

\section{$\$ 34$. Dependence of the Rate on Concentrations.}

We see from Eq. (24.9) that the rate of the heterogeneous elementary reaction varies proportional to $p^{\delta^{I}}$ or, as remarked in $\$ 25$, to $\stackrel{i}{I}\left(N^{\delta_{i}^{I}}\right)^{\nu} i_{i}^{I}$ of the initial complex in the ideal homogeneous fluid provided $\boldsymbol{\theta}_{\sigma^{*}(0)}$ in Eq. (24.9) sufficiently. approximates unity and hence $q_{o}^{\delta_{*}^{*}}$ remains constant owing to the absence of interaction.

In the other extreme case when $\theta_{\sigma^{*}}\left(\delta^{*}\right) \div 1$ or the surface is practically covered by adsorbed molecules $\delta^{M}$ 's, $q_{0^{*}}^{\delta^{*}}$ may be taken corfstant, $\delta^{*}$ being

(1) Guggenheim ; Trans. Faraday Soc. 33 (1937), 607.

(2). Evans and Polanyi ; Trans. Faraday Soc. 33 (1937), 448. 
A Method of Statistical Mechanical Treatment of Equilibrium and Chemical Reactions.

surrounded by definite sort of molecules. The dépendence of the rate on $N_{i}^{\delta_{i}^{r}}$ 's as well as on $N^{\delta_{m}^{M} \text { 's }}$ of molecules $\delta_{m}^{M}$ in homogeneous fluid, whose set,

$$
\delta^{M}=\sum_{m} \nu_{m}^{M} \delta_{m}^{M}
$$

is in equilibrium with adsorbed $\delta^{M}$, is deduced as follows.

Rewriting $\Theta_{o *(1)}$ according to Eq. (2.4) in the form,

$$
\Theta_{\sigma *(0)}=\frac{\mathfrak{D} C_{\sigma *(0)}^{\prime}}{\mathfrak{D} C_{0}^{\prime}}=\frac{\mathfrak{D} C_{\sigma^{*}}(0)}{\mathfrak{D} C_{\sigma^{*}(\delta M)}^{\delta^{M}}} \cdot \frac{\mathfrak{D} C_{\sigma^{*}\left(\delta^{M M}\right)}^{\delta^{M}}}{\mathfrak{D} C_{0}^{\delta^{M}}} \cdot \frac{\mathfrak{D} C_{0}^{\delta^{M}}}{\mathfrak{D} C_{0}^{\prime}}
$$

and noting the relations Eqs. (2.1), (2.2) and (2.3) that,

we have

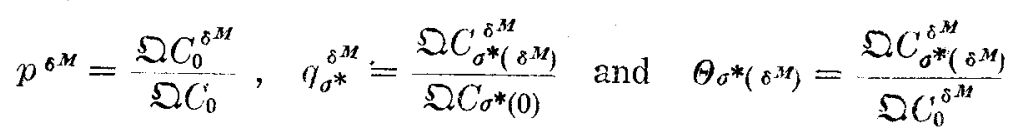

$$
\Theta_{\sigma^{*}(0)}=\frac{p^{\delta^{M L}}}{q_{\sigma^{*}}^{\delta^{M}}} \Theta_{\sigma^{*}}\left(\delta^{M}\right)
$$

or substituting $\Theta_{\sigma *(0)}$ from (34.2) in Eq. (24.9),

$$
\vec{c}_{1}=\kappa \frac{k T}{h} G_{1} \frac{q_{\sigma^{*}}^{\partial^{*}}}{q_{\sigma^{*}}^{\delta M}} \frac{p^{\delta M}}{p^{\delta I}} \Theta_{\sigma^{*}(\delta M)}
$$

We see now that if $\Theta_{\sigma}{ }^{*}\left(\delta^{M}\right)=1$, ${ }_{1}^{*}$ varies proportional to $p^{\delta^{M}} / p^{\hat{o}^{T}}$. i.e.

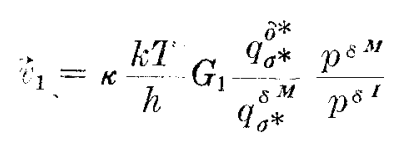

It follows that (I) the rate is proportional to $\left(N^{\delta !}\right)^{\prime \prime} i$ in the ideal homogeneous fluid according to Eqs. (24.2.I) and (5.5), and inversely proportional to $\left(N^{\delta^{M}}\right)^{\nu \frac{M}{m}}$ there.

If in latter case (II) it happens that $\delta_{i}^{\prime}$ 's and $\delta_{m}^{M}$ 's are individually identical to each other or that the initial complex $\delta^{I}$ itself exclusively occupies $\sigma^{*}$, the rate is independent of the concentration $N^{\delta_{i}^{1}}$ 's or of $N^{\delta M}$ 's. If on the other hand (III) $\delta_{i}^{l}$ 's are partially identical to $\delta_{m}^{M}$ 's the 
conclusions (I) holds true with the remaining part of $\delta_{i}^{I}$,s and $\delta_{m}^{M}$,s.

The above reasoning about the effect of $p^{\delta^{M}}$ or $\stackrel{m}{I I}\left(N^{\delta_{m}^{M}}\right)^{\nu} \frac{M}{m}$ on the rate affords the theory of the catalytic poison. Rather curious conclusion that the rate is inversely proportional to $\stackrel{m}{I}\left(N^{\delta^{M}}\right)^{\nu}{ }^{\frac{M}{m}}$ of $\delta^{M}$, even when the latter nearly covers the catalyst, is correctly arrived at by Laidler, Glasstone and Eyring(1), who extended their theory treating the heterogeneous elementary reaction like a homogeneous one between cites and molecules.

\section{$\$ 35$. The Tewloratuge Lariation of the Rate.}

The temperature variation of $\vec{i}$ of the heterogeneous elementary reaction of the effusion type $e^{(*)}$ is expressed according to $\mathrm{Eq} \cdot(24.9 . \vec{v})$, as that.

$$
R T^{:} \frac{\partial \log \vec{v}_{1}}{\partial T}=R T+\left.\Delta\right|^{*} \bar{E}_{o *}+\left.\Delta\right|_{\sigma *(0)} ^{0} \bar{E}
$$

where,

$$
\begin{aligned}
& \Delta_{i}^{*} \bar{E}_{\sigma *}=\Delta_{\sigma *(\delta *)}^{\alpha} \bar{E}-\Delta_{\delta}^{p} I \bar{E}, \quad d_{\sigma^{*}(0)}^{\theta} \bar{E}=R T^{*} \frac{\partial \log \theta_{\sigma *(0)}}{\partial T} \\
& \Delta_{\sigma *(\delta *)}^{\alpha} \bar{E}=R T^{*} \frac{\partial \log q_{\sigma *}^{\delta^{*}}}{\partial T} \quad \text { and } \quad \Delta_{\delta}^{p} I \bar{E}=R T^{*} \frac{\partial \log p^{\delta^{l}}}{\partial T}
\end{aligned}
$$

$\Delta_{i^{*}(0)}^{\beta} \bar{E}, \Delta_{\sigma *\{\delta *)}^{q} \bar{E}$ and $\Delta_{\delta}^{p} I \bar{E}$ are according to $\mathrm{Eq} .(7.6)$ increments due to the process respectively associated with $\Theta_{\sigma *(0)}, q^{\delta^{*}}$ and $p^{\delta^{x}}$, of the average energy $\bar{E}$ or of the internal energy of the whole assembly, whose external parameters are fixed. $\left.\Delta\right|^{*} \bar{E}_{\sigma *}$ is hence according to Eq. (35.2) the increment caused by bringing $\delta^{I}$ from somewhere in the assembly into preliminarily evacuated $\sigma^{*}$ to form $\delta^{*}$ there, the $d /$ 's are thus increments. of internal energy or of enthalpy according as the assembly of interest is $A_{V}$ or $A_{r}$ respectively(**).

The alternative expression of the temperature variation is obtained by differentiating $\mathrm{Eq}$. (34.3) as that,

(1) Laidler, Glasstone \& Eyring; J. Chem. Phys. \& (1940), 659, 667.

(*) See p. 57.

(**) Cf, $\$ 13$. 
A Method of Siatistical Mechanical Treatment of Equilibinim and Chemical Reactions.

$$
R T^{*} \frac{\partial \log \vec{\imath}_{1}}{\partial T}=R T+A^{*} \bar{E}_{\sigma^{*}}+\Delta I^{M} \bar{E}_{\sigma^{*}}+\Delta \Delta_{\sigma^{*}\left(\delta^{M}\right)}^{\mathrm{a} \bar{E}^{*}}
$$

where

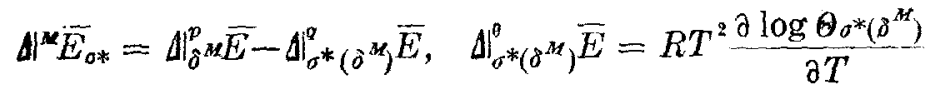

$$
\begin{aligned}
& (35.5),(35.6 . \theta) \\
& \Delta_{\delta}^{p} \Sigma \bar{E}=R T^{z} \frac{\partial \log p^{\delta^{M}}}{\partial T} \text { and } A_{\sigma^{*}\left(\delta^{M}\right)}^{\alpha} \bar{E}=R T^{2} \frac{\partial \log q_{\sigma^{*}}^{\delta^{* *}}}{\partial T} \\
& \text { (35.6.p), (35.6. } q)
\end{aligned}
$$

The latter three quantities are, according to $\mathrm{Eq}$. (7.6) increments due

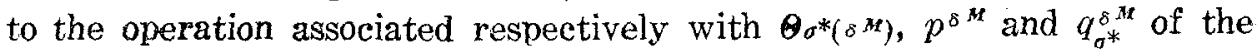
average energy $\bar{E}$ or the internal energy of the whole assembly whose external parameters are fixed. $\Delta \mathbb{H}^{M} \bar{E}_{0^{*}}$ is similarly as in the previous case such increment caused by bringing $\delta^{M}$ from somewhere in the assembly into $\sigma^{*}$. These increments are those of the internal energy or of the enthalpy of the interested assembly according as the latter is Av or $A_{P}^{(*)}$.

If practically all of $\delta^{A}$ 's are situated in the homogeneous part of the assembly, $\| \mathbb{E}^{\pi} \bar{E}_{0^{*}}$ is identified with the heat of adsorption at constant. volume or pressure respectively according as the assembly of interest is either $A_{V}$ or $A_{P}$.

In the extreme case when $\theta_{\sigma^{*}(0)}=1$ or $\theta_{\sigma^{*}\left(\delta^{M}\right)}=1, \Delta_{\sigma^{*}(0)}^{\theta_{0}} \bar{E}$ or $\Delta_{\sigma^{*}\left(\hat{o}^{M}\right)}^{\theta^{M}} \bar{E}$ respectively vanishes according to $\mathrm{Eq} .(35.3 . \theta)$ or $(35.6 . \theta)$ and hence Eq. (35.1) or (35.4) for $R T^{2} \frac{\partial \log \vec{v}_{1}}{\partial T}$ assumes respectively the form,

$$
\begin{aligned}
& R T^{2} \frac{\partial \log \vec{v}_{1}}{\partial T}=R T+H^{*} \widetilde{E}_{\sigma^{*}} \\
& \text { or } \\
& K T^{*} \frac{\partial \log \vec{v}_{1}}{\partial T}=R T+\Delta i^{*} \widehat{E}_{\sigma^{*}}+\Delta \|^{M} \widehat{E}_{\sigma^{*}}
\end{aligned}
$$

As to the relative magnitude of $R T^{2} \frac{\partial \log \vec{v}_{1}}{\partial T}$ at the both extremities

(*) Cf. $\$ 13$. 
and to the temperature region of their appearance, the following may be inferred assuming the relation of $\mathrm{Eq} .(17.2)^{(*)}$

$$
\theta=\Theta_{\sigma}^{*}\left(\delta^{s t}\right)=1-\Theta_{\sigma *(0)}
$$

Equating alternative forms of $R T^{2} \frac{\partial \log \vec{v}_{1}}{\partial T}$, Eq. (35.1) and Eq. (35.4), with due regard to Eqs. $(35.3 . \theta)$, (35.5) and $(35.6 . \theta)$, we have,

$$
R T^{2} \frac{\partial \log \theta_{o *(0)}}{\partial T}-R T^{2} \frac{\left.\partial \log \theta_{\sigma^{*}\left(\delta^{*}\right)}\right)}{\partial T}=\Delta T^{M} \bar{E}_{o *}
$$

or by Eq. (35.8)

$$
R T^{2} \frac{\partial \log (1-\theta)}{\partial T}-R T^{2} \frac{\partial \log \theta}{\partial T}=\left.\Delta\right|^{M} \bar{E}_{\sigma *}
$$

Eq. (35.10) shows, since $-R T^{2} \frac{\partial \log \theta}{\partial T}$ and $R T^{\prime 2} \frac{\partial \log (1-\theta)}{\partial T}$ are necessarily of equal sign, that $\theta$ increases or decreases with increasing temperature according as $\left.\Delta\right|^{M} \bar{E}_{\sigma *}$ is negative or positive respectively. Provided that the latter is at least approximately constant, the extreme cases $\theta=0$ and $\theta=1$ should hence respectively appear at lower and higher extremities of temperature or reverse according as $\left.A\right|^{M} \bar{E}_{o *}$ is negative or positive, as shown by the scheme,

\begin{tabular}{c|cc}
$\Delta \|^{n} \bar{E}_{\sigma^{*}}$ & $\theta \rightarrow 0$ & $\theta \rightarrow 1$ \\
\hline neg. & Temp. lower & Temp. higher \\
pos. & Temp. higher & Temp. lower
\end{tabular}

On the other hand Eqs. (35.7.1) and (35.7.I) show that $R T^{2} \frac{\partial \log \vec{v}_{1}}{\partial T}$ $=E_{H}$ is greater at $\theta=0$ than at $\theta=1$ or reversed according as $\|^{M} \bar{E}_{\text {o* }}$ is negative or positive as shown by the scheme,

(*) This holds true when the states $\sigma^{*}(0)$ and $\sigma^{*}\left(\delta^{*}\right)$ are practically only possible alternative states of $\sigma^{*}, \Theta_{\sigma^{*}}\left(\sigma^{*}\right)$ being thereby negligible. Cf. $\$ 17$. 
A Method of Statistical Mechanical Treatment of Equilibriam and Chemical Reactions.

\begin{tabular}{c|cc}
$\Delta^{M} \widehat{E}_{\mathrm{o} *}$ & $\theta \rightarrow 0$ & $\theta \rightarrow 1$ \\
\hline neg. & $E_{H}$ greater & $E_{H}$ smaller \\
pos. & $E_{H}$ smaller & $\cdot E_{H}$ greater
\end{tabular}

We see readily, overlapping above two schemes, that $E_{H}$ is always greater at the lower extremity of temperature than at the higher.

$\log \vec{\imath}_{1}$ may hence vary linearly with $1 / R T$ respectively at the extremities, the inclination gradually decreasing over the transcient region by the amount $|\Delta|^{s} \bar{E}_{\sigma^{*}} \mid$ with increasing temperature. In the case when $\left.\Delta\right|^{*} \bar{E}_{\text {o* }}$ and $\Delta{ }^{x} E_{o *}$ differ in sign and $\left.|\Delta|^{*} \bar{E}_{\text {o* }}|<| \Delta\right|^{x} \bar{E}_{0 *} \mid$, the rate will have a maximum or an optimum temperature in the transcient region. If we exclude the possibility that $\Theta \sigma^{*}(\delta M)=1$ at higher extremity of temperature ${ }^{(*)}$, only possible ease is that $\Delta^{M M^{M}} \bar{E}_{\sigma^{*}}>0$ and $\theta_{\sigma^{*}}\left(\hat{o}^{M}\right)=0$ or 1 respectively at higher or lower extremity of temperature.

The two extreme cases corresponding to Eq. (35.7) are recongnized by Hinshelwood ${ }^{(1)}$ who treats the heterogeneous elementary reaction in extension of the theory of homogeneous one, in terms of "the rate constant $k^{\prime}$ " and of "the apparent heat of activation $R T^{2} \frac{\partial \log k^{\prime}}{\partial T}$ of the heterogeneous reaction." Hinshelwood arrives by several assumptions and approximations at the relation for the case $\theta=0$ that the "apparent heat of activation, $R T^{2} \frac{\partial \log k^{\prime}}{\partial T}$ " equals "the true heat of activation" minus "the energy of desorption." The "true heat of activation" is according to him the heat required to bring up an adsorbed reaction complex to the critical complex and the "energy of desorption" the hert required to set the adsorbed reaction complex

(*) Provided that ${ }^{M}$ forms a single molecule in the horrogeneous fluid, we have according to Egs. (17.5) and (35.10),

$$
f^{M} \bar{E}_{\sigma *}=N_{A} \varepsilon_{\sigma}
$$

Eq. (17.5) shows on the other hand that $\theta$ increases with increasing temperature, when ${ }^{M}{ }^{M} E_{\sigma} *$ or $\varepsilon_{0}$ is negative, but $\theta$ may approximate unity only when $|\sigma| N^{\delta^{M}} \gg 1$ or $\delta^{M}$ in the homogeneous fluid is far more concentrated than at saturation on the boundary, a situation which can hardly be realized.

(1) "Kinetics of Chemical Change in Gaseous System" Oxford (1923), 354: 


\section{Journal of the Research Institute for Catalysis}

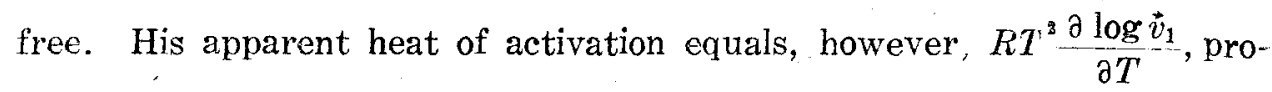
vided that the " rate constant" is determined at constant $N^{\delta_{i}^{I}}$ 's throughout a range of temperature as is usually done, since he defines the "rate constant" as the ratio of the rate to $\stackrel{i}{I}^{(}\left(N^{\delta}{ }_{i}^{I}\right)^{\nu I}$ in a homogeneous fluid. Hence $R T^{2} \frac{\partial \log k^{\prime}}{\partial T}$ or $R T^{2} \frac{\partial \log \vec{v}_{1}}{\partial T}$ at $\theta=0$, which equals except the minor term $R T^{(*)}$ the increment of $\bar{E}$ or of $U$ caused by bringing the initial complex to the critical state in $\sigma^{*}$ according to Eq. $(35.7 .1)^{(* *)}$, may simply be divided, if one prefers as did by Hinshelwood, in two parts i.e. that due to the process of bringing the initial complex simply into $\sigma^{*}$ or negative "energy of desorption" and that of raising the initial complex there to the critical complex provided that initial complexes are situated practically exclusively in the homogeneous fluid.

For the case when $\theta \sigma^{*}\left(\delta^{M}\right)=1$ Hinshelwood similarly arrives at an expression equating $R T^{3} \frac{\partial \log k^{\prime}}{\partial T}$ to the sum of the "apparent heat of activation" and the "energy of desorption" of " petarding gas." This conforms again with Eq. (35.7.II) since the "energy of desorption" of the "retarding gas" exactly corresponds to $\left.\Delta\right|^{M} \bar{E}_{0 *}$ of $\delta^{N}$.

We see, however, no advantage to force the rate constant method of the homogeneous elementary reaction into the heterogeneous reaction, where the "rate constant" is in general by no means a constant, and to detail the picture so that the initial complex preliminarily assumes the state what is called adsorbed at $\sigma^{*}$ before making the critical complex there. We should rather, specialize the use of the rate constant and its temperature variation duly to the homogeneous elementary reaction and

(*) Since the statistical average of kinetic energy of the mode of motion normal to the critical surface amounts just to $R T$ if reckoned per mol of the critical complex [Tem* kin; Acta Physicochim. U.R.S.S. 13 (1940), 733.], the right hand side of Eq. (1.7.I) gives the increment of $\bar{E}$ reckoned per mol of reacting complex caused by bringing the initial over into the critical complex transiting the critical surface.

(**) If $\theta \neq 0$ or $\sigma^{*}$ is not preliminarily evacuated with certainty, the latter process must be associated with the more or less additional increment for clearing up $\sigma^{*}$ which amounts as much as to $A^{M} \bar{E}_{\sigma *}$ in the extreme case of $\mathrm{Eq}$. (35.7.1I) when $\theta=1$. 
deal directly with $\vec{v}_{1}$ and $R T^{2} \frac{\partial \log \vec{v}_{1}}{\partial T}$ without introducing the intermediate state, which may even be fictitious.

\$36. The Rate Expression Allowed for the Mutual Interaction among Adsorbed Molecules.

We will now formulate the rate of the heterogeneous elementary reaction allowing for the mutual interaction among adsorbed molecules with an example of the catalytic atomization of hydrogen on the surface of a metallic catalyst.

Our assembly will consist of a metallic catalyst in the hydrogen atomosphere; where the catalysed dissociation of hydrogen molecules is going on, each of them changing into a pair of adsorbed hydrogen atoms. Metal atoms of our catalyst are allayed as in $\$ 20$ on a plane square pattern, each affording one of identical cites $\sigma$ 's for an adsorbed hydrogen atom whereas each adjascent pair proving $\sigma^{*}$ for the critical complex $H_{2}^{*}$, which consists of two hydrogen atom. .

Expressing $p^{s}$ of the initial complex $\delta^{I}$ according to Eq. (5.5) in the form,

$$
p \delta^{l}=\frac{Q^{H_{z}}}{N^{H_{2}}}
$$

and $p^{h^{\prime}}$ of $\delta^{r}$, which consist of two hydrogen atoms, according to Eq. (4.1) in the form.

$$
p^{I}=\left(p^{H}\right)^{2}
$$

we have according to Eq.(24.9)

$$
\begin{aligned}
& \ddot{\iota}_{1}=\kappa \frac{k T}{h} G_{1} \cdot q_{\sigma^{*}}^{H^{*} \Theta_{o^{*}(0)}} \frac{N^{H_{a}}}{Q^{H_{2}}} \\
& \check{t}_{1}=\kappa \frac{k T}{h} G_{1} q_{\sigma^{*}}^{H_{2}^{*}} \Theta_{0^{*}(0)}\left(p^{H}\right)^{-2}
\end{aligned}
$$

These expressions for the rate will be developed into the form accessible to the numerical calculation taking the mutual interaction among adsorbed molecules into account, but neglecting that between latters and molecules in the homogeneous fluid and assuming that any cite $\sigma$ is either ocsupied by $H$ or else vacant i.e. that, 


$$
\theta=\Theta_{\sigma(H)}=1-\Theta_{o(0)}
$$

For the first approximation it is assumed that the additional reversible work ${ }^{*)}$ required to bring up a hydrogen atom or the critical complex to a cite due to surrounding adsorbed hydrogren atoms is proportional to their degree of adsorption $\theta$, i.e. that.

and

$$
\begin{aligned}
& -R T \log q_{o}^{H}=-R T \log q_{\sigma, 0}^{H}+\theta \omega \\
& -R T \log q_{\sigma^{*}}^{H_{2}^{*}}=-R T \log q_{o^{*}, 0}^{H_{2}^{*}}+\theta \omega^{*}
\end{aligned}
$$

where $-R T \log q_{\sigma, 0}^{H}$ or $-R T^{\prime} \log q_{\sigma^{*}, 0}^{H_{2}^{*}}$ is the reversible work reckoned per mol required, in the absence of interaction, to bring up a hydrogen atom or a reaction complex from its standard state into $\sigma$ forming an adsorbed hydrogen atom or a critical complex respectively there, $\theta \omega$ or $\theta \omega^{*}$ being the additional reversible work due to the interaction taken proportional to $\theta$.

Assuming further that,

$$
\Theta_{\circ} *_{(0)}=(1-\theta)^{2}
$$

we have from Eqs. (36.5. $\left.\mathrm{H}_{2}^{*}\right)$, (36.6) and (36.3.t) that,

$$
\vec{\nabla}_{1}=\kappa \frac{k T}{h} G_{1} \frac{q_{\sigma *}^{H_{2}^{*}}}{Q^{H_{2}}} e^{-\frac{\theta \omega *}{R T}} N^{H_{2}}(1-\theta)^{2}
$$

Eq. (36.7) gives the rate as a function of $\theta$ and $N^{3 F_{9}}$.

Expressing $p^{H}$, on the other hand, in the form,

$$
p^{H}=\frac{1-\theta}{\theta} q_{o}^{H}
$$

according to Eqs. (5.1) and (36.4), we have, by Eqs. (36.3.v), (36.5. $\left.\mathrm{H}_{2}^{*}\right)$, (36.6) and (36.8) for the rate $\hat{v}$ of the reverse elementary reaction that,

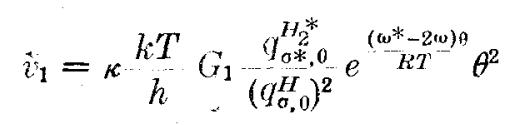

The reverse rate is thus a function solely of $\theta$.

(*) Cf. $\$ 7$. 
The above procedure of allowing for the mutual interaction is applied to the theory of hydrogen electrode process on nickel, with the ratedetermining step of the catalysed atomization of hydrogen by Okamoto, Horiuti and Hirota ${ }^{(1)}$, who arrive at semi-quantitative explanation of the process especially of Tafel's empirical rule ${ }^{(2)}$.

We now ealculate the rate excluding the latter approximations but assuming instead that the interaction between hydrogen atoms including those of critical complex is significant only between directly neighbouring ones.

We consider our assembly $C_{\simeq(0)}$ at a particular state, that a group $\sum$ of cites consisting of particular $\sigma^{*}$. and its direct neighbours shown enclosed by full lines in Fig. 1, are unoceupied. The cites belonging to $\Sigma$ is numbered as shown by annexed figures in Fig. 1 .

We now proceed to calculate, with re-

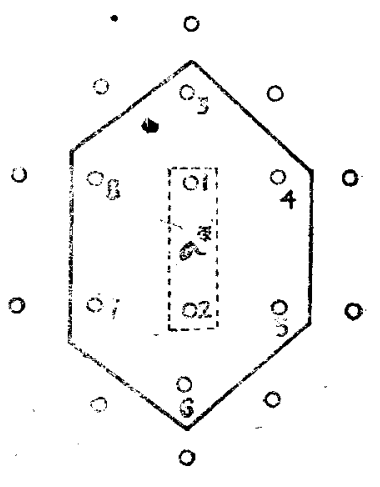

Fig. 1. ference to $\mathfrak{Q} C_{\Sigma(0)}, q_{\sigma^{*}}^{\delta *} \boldsymbol{\theta}_{o *(0)}$ in Eqs. (36.3.3. and (36.3. $\left.\dot{v}_{1}\right)$ which is expressed according to Eqs. (2.2) and (2.4) as that,

$$
q_{o^{*}}^{H_{*}^{*}} \theta_{o *(0)}=\frac{\mathfrak{S} C_{0_{2}^{*}}^{H_{*}^{*}}}{\mathfrak{S} C_{2}^{*}}
$$

$\mathcal{Q} C_{{ }^{*}\left(H_{2}^{*}\right)}^{H_{2}^{*}}$ is given similarly as in $\S 20$, by

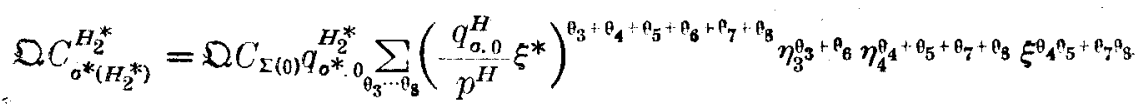

where $\eta$ 's of the cites $\sigma_{3}$ and $\sigma_{6}$ on the one hand, and of $\sigma_{4}, \sigma_{5}, \sigma_{7}$ and $\sigma_{8}$ on the other hand are taken respectively identical with each other; because of the symmetry of the figure.

$Q C_{0}$ is given by, 233.

(1) Okamóto, Horiuti and Hirota; Se. Pap. Inst. Phys. Chem. Res. Tokio, 28 (1936),

(2) Tafel; Z. physik. Chem., 50 (1905), 641. Cf. Bowden and Agar; Annual Report, Chem. Soc. London 35 (1939), 90. 


$$
\begin{aligned}
& \text { Journal of the Research Ins'itute for Catalysis }
\end{aligned}
$$

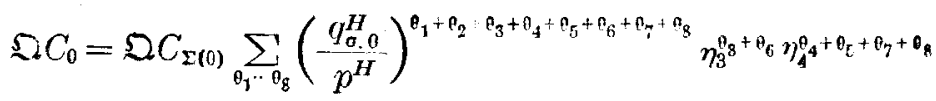

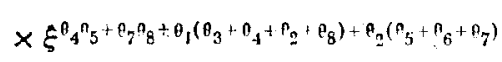

By Eqs. (36.10), (36.11) and (36.12) we have now,

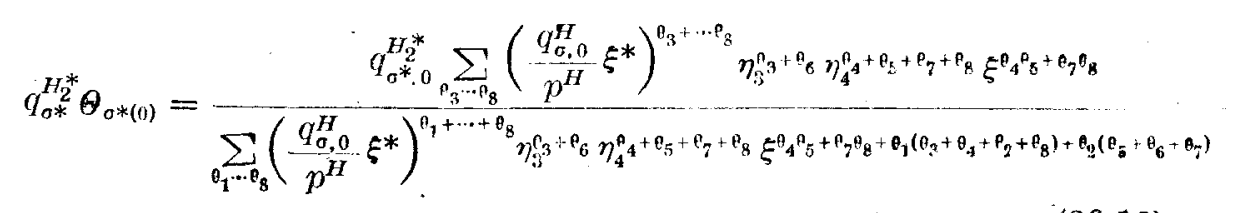

For unknown constants $\eta_{3}$ and $\eta_{4}$ we have on the other hand the relation,

$$
\mathfrak{Q} C_{\sigma_{1}(0)}=\mathfrak{Q} C_{\sigma_{3}(0)}=\mathfrak{Q} C_{\sigma_{4}(0)}
$$

as in $\S 20$, because of physical identity of $\sigma_{1}, \sigma_{3}$ and $\sigma_{1}$.

$\mathfrak{D C} C_{\sigma_{1}(0)}, \mathfrak{D} C_{\sigma_{3}(0)}$ or $\mathfrak{Q} C_{\sigma_{4}(0)}$ is respectively obtained by putting $\theta_{1}, \theta_{3}$ or $\theta_{4}$ in Eq. (36.12) at zero.

$S C_{0_{1}(0)}$ in Eq. (36.14) and $\mathfrak{a C}$ in Eq. (36.12) are on the other hand related to $\theta$ according to Eqs. (2.4) and (36.4) by,

$$
1-\theta=\frac{\mathfrak{N} C_{a_{1}(0)}}{\mathfrak{S C} C_{0}}
$$

Substituting now $q_{o^{*}}^{H_{2}^{*}} \theta_{\sigma^{*}(0)}$ from Eq. (36.13) into Eq. (36.3. $\left.\hat{\imath}_{1}\right)$ or (36.3.v) and eliminating $p^{H}, \eta_{3}$ and $\eta_{4}$ therefrom by Eqs. (36.14) and (36.15), we have the expression for $\vec{v}_{1}$ as the function of $N^{H_{0}}$ and $\theta$ or that for $\dot{v}_{1}$ as the function of $\theta$ respectively.

Remembering now two of cites are occupied anew as the result of one act of the catalysed dissociation, the over-all increasing rate of adsorbed hydrogen atoms is given by

$$
G_{1} \frac{d \theta}{d t}=2\left(\vec{\imath}_{1}-\hat{v}_{1}\right)
$$

or by integration,

$$
\begin{gathered}
\frac{G_{1}}{2} \int_{v_{1}}^{r_{2}} \frac{d \theta}{\hat{v}_{1}-\bar{v}_{1}}=t_{2}-t_{1} \\
-78-
\end{gathered}
$$


A Method of Statistical Mechanical Treatment of Equitibrium and Chemical Reactions.

where $\theta_{1}$ and $\theta_{2}$ are respectively the degree of adsorption at the time $t_{1}$ and $t_{2}$. Substituting $\vec{v}_{1}$ and $\hat{v}_{1}$ of either approximation and estimating $\kappa, G, q_{\sigma *, 0}^{H_{2}^{*}}, q_{\sigma, 0}^{H}, \omega^{*}, \omega, \xi^{*}$ and $\xi$ properly, we have the relation betiveen $\theta$ and $t$ accessible to the experimental verification.

Actual calculation and iț experimental verification will be presented in a later paper. 